\title{
On the supersymmetry invariance of flat supergravity with boundary
}

\author{
Patrick Concha, ${ }^{a}$ Lucrezia Ravera $^{b}$ and Evelyn Rodríguez ${ }^{c}$ \\ ${ }^{a}$ Instituto de Física, Pontificia Universidad Católica de Valparaíso, \\ Casilla 4059, Valparaiso, Chile \\ ${ }^{b}$ INFN, Sezione di Milano, \\ Via Celoria 16, I-20133 Milano, Italy \\ ${ }^{c}$ Departamento de Ciencias, Facultad de Artes Liberales, Universidad Adolfo Ibáñez, \\ Viña del Mar, Chile \\ E-mail: patrick.concha@pucv.cl, lucrezia.ravera@mi.infn.it, \\ evelyn.rodriguez@edu.uai.cl
}

ABSTRACT: The supersymmetry invariance of flat supergravity (i.e., supergravity in the absence of any internal scale in the Lagrangian) in four dimensions on a manifold with non-trivial boundary is explored. Using a geometric approach we find that the supersymmetry invariance of the Lagrangian requires to add appropriate boundary terms. This is achieved by considering additional gauge fields to the boundary without modifying the bulk Lagrangian. We also construct an enlarged supergravity model from which, in the vanishing cosmological constant limit, flat supergravity with a non-trivial boundary emerges properly.

Keywords: Supergravity Models, Differential and Algebraic Geometry

ArXiv EPrint: 1809.07871 


\section{Contents}

1 Introduction 1

2 Geometric approach and supersymmetry invariance in presence of a boundary

3 Flat supergravity in presence of a non-trivial boundary 4

3.1 Maxwell supersymmetry 9

4 Supergravity with generalized cosmological constant in presence of a nontrivial boundary

4.1 Rheonomic construction of the bulk Lagrangian 11

$\begin{array}{lll}4.2 & \text { Supersymmetry invariance in presence of a boundary } & 14\end{array}$

$\begin{array}{lll}\text { 4.2.1 AdS-Lorentz supersymmetry } & 17\end{array}$

$\begin{array}{lll}5 & \text { Discussion } & 19\end{array}$

\section{Introduction}

The presence of a boundary in (super)gravity theories has been of particular interest in the last 40 years [1-4]. The addition of boundary terms plays an important role in the so-called AdS/CFT duality [5-11]. Such duality between a quantum field theory living on the boundary and a string theory on asymptotically AdS spacetime implies in the supergravity limit a one to one correspondence between fields of the bulk supergravity theory and quantum operators in the boundary CFT. This requires to consider proper boundary conditions for the supergravity fields which act as sources for the operators of the CFT. Interestingly, the divergences of the bulk metric can be cancelled by adding appropriate counterterms at the boundary (holographic renormalization) [12-19]. More recent results of proper counterterms in gravity theories beyond Einstein theory have been developed in [20-23].

The inclusion of boundary terms in supergravity has been studied by diverse authors in [24-40]. In particular, in [26, 27, 30, 31] it was pointed out that, unlike the GibbonsHawking prescription [2], the supersymmetry invariance of a supergravity action should be satisfied without imposing Dirichlet boundary conditions. Interestingly, for the $\mathcal{N}=1$ three-dimensional supergravity, it was proven that the boundary term reproduces not only the Gibbons-Hawking-York boundary term but also the counterterm allowing to regularize the action [31].

Recently, the authors of [33] have shown that the supersymmetry invariance of $\mathcal{N}=1$ and $\mathcal{N}=2$ four-dimensional supergravity with cosmological constant requires the presence 
of topological terms. Such result can be seen as the supersymmetric extension of those obtained in AdS gravity in which the addition of the topological Gauss-Bonnet term allows to regularize the action [41-45]. Subsequently, in [35, 40], using the same geometric approach used in [33], it was shown that the supersymmetric extension of a Gauss-Bonnet like gravity is required to restore the supersymmetry invariance of enlarged supergravities in the presence of a non-trivial boundary. ${ }^{1}$ Interestingly, the full supergravity actions obtained in $[33,35,40]$ can be rewritten in terms of the super curvatures à la MacDowellMansouri [46].

Boundary conditions imposed at a finite value of the radial coordinate have been studied in $D=4$ supergravity with zero cosmological constant in $[26,28,29]$. However, the limit case of vanishing cosmological constant in the presence of a non-trivial boundary remains poorly explored. In particular, the flat supergravity Lagrangian is not supersymmetric when a non-trivial boundary is considered and, on the other hand, a flat limit cannot be naively applied to the MacDowell-Mansouri Lagrangian since it reduces only to boundary terms:

$$
\mathcal{L}=\epsilon_{a b c d} \mathcal{R}^{a b} \mathcal{R}^{c d}+D \bar{\psi} \gamma_{5} D \psi
$$

This can be directly seen from the super curvatures $\mathcal{R}^{a b}$ and $D \psi$ of the Poincare superalgebra which do not allow the presence of the Einstein-Hilbert (EH) neither the Rarita Schwinger (RS) terms in the Lagrangian. This inconvenient appears only in presence of a boundary since the bulk flat Lagrangian can be recovered directly from the bulk OSp $(4 \mid 1)$ supergravity as a vanishing cosmological constant limit.

Let us specify that, here as well as in the sequel of this work, with "flat supergravity" we mean supergravity in the absence of any internal scale in the Lagrangian.

In this paper, we restore the supersymmetry invariance of the four-dimensional flat supergravity in presence of a non-trivial boundary. To this purpose, we introduce appropriate boundary terms to the Lagrangian such that the supersymmetry is recovered. This is achieved by adding new bosonic and fermionic gauge fields to the boundary in addition to the usual spin-connection, vielbein and gravitino. Interestingly, we find that the boundary values of the super curvatures are fixed by the field equations of the full Lagrangian (understood as bulk plus boundary contributions). In particular, the full Lagrangian obtained can be rewritten in terms of super curvatures of a particular superalgebra known as Maxwell superalgebra [47]. We also present a proper vanishing cosmological constant limit from an enlarged supergravity theory with a non-trivial boundary. Such enlarged supergravity Lagrangian can be rewritten à la MacDowell-Mansouri for a deformation of the $\mathfrak{o s p}(4 \mid 1)$ superalgebra which corresponds to a new supersymmetric extension of the AdS-Lorentz algebra [48, 49].

The paper is organized as follows: in section 2, we present a brief review of the geometric approach we will adopt for the formulation of supergravity in superspace. The supersymmetry invariance of a supergravity Lagrangian in the presence of a non-trivial

\footnotetext{
${ }^{1}$ In presence of a non-trivial boundary of spacetime, that is when the boundary is not at infinity, the fields do not asymptotically vanish, and this has some consequences on the invariances of the theory, in particular on supersymmetry invariance.
} 
boundary is explicitly discussed within this framework. Section 3 and 4 contain our main results. In particular, in section 3 we explore the supersymmetry invariance of flat supergravity in presence of a non-trivial boundary, while in section 4 we construct an enlarged supergravity Lagrangian with a generalized cosmological constant term using the geometric approach. We show that the flat limit can be appropriately applied in presence of a boundary. We conclude our work with some comments and possible future developments.

\section{Geometric approach and supersymmetry invariance in presence of a boundary}

An interesting and powerful approach for constructing supergravity theories is the geometric or rheonomic approach [50]. The principal demand of any supergravity theory is the invariance of the action under supersymmetry transformations. In the rheonomic approach, a supergravity theory is given in terms of 1-form superfields defined on superspace (whose basis is given by the supervielbein), and the supersymmetry transformations on spacetime correspond to diffeomorphisms in the fermionic directions of superspace. Thus, the principle of rheonomy makes the extension from spacetime to superspace uniquely defined and consequently allows for a geometric interpretation of the supersymmetry rules.

In this framework, the supersymmetry invariance of the Lagrangian is expressed by the vanishing of the Lie derivative of the Lagrangian for infinitesimal diffeomorphisms in the fermionic directions, that is to say

$$
\delta_{\epsilon} \mathcal{L}=l_{\epsilon} \mathcal{L}=\imath_{\epsilon} d \mathcal{L}+d\left(\imath_{\epsilon} \mathcal{L}\right)=0
$$

From condition 2.1 it is direct to see that when a supergravity Lagrangian is considered on spacetimes without boundary, it implies that $\left.\imath_{\epsilon} \mathcal{L}\right|_{\partial \mathcal{M}}=0$. Then, in the absence of a (non-trivial) boundary, $\imath_{\epsilon} d \mathcal{L}=0$ results to be a sufficient condition for supersymmetry invariance. Nevertheless, when the background spacetime has a non-trivial boundary, the condition $\left.\imath_{\epsilon} \mathcal{L}\right|_{\partial \mathcal{M}}=0$ becomes non-trivial. Thus, in order to verify the invariance of the Lagrangian in presence of a non-trivial boundary, it is necessary to check it explicitly (see [33] for further details).

As it is well explained in [50], in the geometric approach the 1 -forms fields $\mu^{A}$ are extended from spacetime to superspace, such that the mapping $\mu^{A}(x) \rightarrow \mu^{A}(x, \theta)$ is defined by rheonomy, which amounts to the following: the superspace equations of motion are given in terms of the superspace curvatures, and can be analyzed by expanding the curvatures along the basis of 2 -forms in superspace,

$$
R^{A}=R_{B C}^{A} \mu^{B} \mu^{C}
$$

expanding the curvature 2 -forms $R^{A}$ in superspace along the supervielbein basis $\left\{V^{a}, \psi\right\}$,

$$
R^{A}=R_{b c}^{A} V^{b} V^{c}+R_{a \alpha}^{A} V^{a} \psi^{\alpha}+R_{\alpha \beta}^{A} \psi^{\alpha} \psi^{\beta},
$$

from the analysis of the equations of motion one finds that the outer components $R_{a \alpha}^{A}, R_{\alpha \beta}^{A}$ of the curvature 2-forms in superspace are expressed, on-shell, as linear tensor combinations 
of the inner components $R_{a b}^{A}{ }^{2}$ From the physical point of view, this means that no new degree of freedom is introduced in the theory other than those already present on spacetime. Thus, rheonomy avoids the introduction of spurious degrees of freedom which would appear in the theory if the outer components of the curvatures were independent fields. Furthermore, if we also assume Lorentz gauge invariance of the rheonomic parametrization for the curvature 2-forms and homogeneous scaling of all the terms involved, then the form of the superspace curvatures is completely determined, except for some constant coefficients which are fixed by the Bianchi identities.

Then, the rheonomic parametrization of the super curvatures can be equivalently read as the on-shell prescription for the contractions of the super field-strengths. On the other hand, one can prove that, in the geometric formalism, the condition for a theory (in particular, for its Lagrangian) to be supersymmetry invariant $\left(\imath_{\epsilon} d \mathcal{L}=0\right)$ is fulfilled if one requires constraints on the components of the curvatures (in particular, on the contractions of the super field-strengths). These requirements turn out to be the same of the on-shell prescription for the contractions of the super field-strengths arising from the rheonomic parametrization of the super curvatures. Thus, one retrieves exactly the same constraints on the curvatures as those found from the equations of motion. Moreover, these constraints provide the supersymmetry transformation laws of the fields on spacetime, under which the spacetime Lagrangian is invariant up to boundary terms. One can then show (see [50] for details) that this restricted form of the curvatures in superspace implies that the supersymmetry transformations leaving the Lagrangian invariant close, in general, only on-shell (that is, transformations close only on the equations of motion). Finally, the rheonomy principle is completely equivalent to the requirement of spacetime supersymmetry (see [50]), the latter being interpreted from the geometrical point of view as the requirement that the superspace equations of motion imply that the outer components of the super curvatures are linearly expressible in terms of the inner components.

Along this work, we will apply the geometric approach to restore the supersymmetry of flat supergravity in the presence of a boundary: in this context, one can introduce in a geometric way appropriate boundary terms to the Lagrangian in such a way that the action, including the boundary contributions, results to be invariant under supersymmetry transformations. In particular, since the geometric approach does not require an off-shell formulation of bulk supergravity, we shall use the on-shell formulation of supergravity (that is, transformations close only on the equations of motion).

\section{Flat supergravity in presence of a non-trivial boundary}

The Lorentz-covariant super field-strengths in four dimensions are

$$
\begin{aligned}
\mathcal{R}^{a b} & \equiv d \omega^{a b}+\omega_{c}^{a} \omega^{c b}, \\
R^{a} & \equiv D V^{a}-\frac{1}{2} \bar{\psi} \gamma^{a} \psi=d V^{a}+\omega_{b}^{a} V^{b}-\frac{1}{2} \bar{\psi} \gamma^{a} \psi, \\
\rho & \equiv D \psi=d \psi+\frac{1}{4} \omega^{a b} \gamma_{a b} \psi,
\end{aligned}
$$

\footnotetext{
${ }^{2}$ The "outer" components are those having at least one index along the $\psi$ direction of superspace, while when the only non-vanishing components are along the bosonic vielbein they are called "inner".
} 
being $\mathcal{R}^{a b}, R^{a}$, and $\rho$ the Lorentz super curvature, the supertorsion, and the gravitino super field-strength 2 -forms, respectively ( $D=d+\omega$ is the Lorentz covariant derivative), and it is well known that the four-dimensional flat supergravity Lagrangian

$$
\mathcal{L}_{\text {bulk }}=\epsilon_{a b c d} \mathcal{R}^{a b} V^{c} V^{d}+4 \bar{\psi} V^{a} \gamma_{a} \gamma_{5} \rho
$$

is simply given by the EH and RS terms, without involving a cosmological constant term. ${ }^{3}$ The field equations read (up to boundary terms)

$$
\begin{aligned}
\epsilon_{a b c d} R^{c} V^{d} & =0 \\
2 \epsilon_{a b c d} \mathcal{R}^{a b} V^{c}+4 \bar{\psi} \gamma_{d} \gamma_{5} \rho & =0 \\
8 V^{a} \gamma_{a} \gamma_{5} \rho+4 \gamma_{a} \gamma_{5} \psi R^{a} & =0 .
\end{aligned}
$$

Here, $a, b, \cdots=0,1,2,3$ are Lorentz indices and $\epsilon_{a b c d}$ is the four-dimensional Levi-Civita tensor. Note that the supergravity Lagrangian scales with $\omega^{2}$, being $\omega^{2}$ the scale-weight of the EH term. In fact, $\left[\omega^{a b}\right]=\omega^{0},\left[V^{a}\right]=\omega^{1}$ and $[\psi]=\omega^{1 / 2}$.

The Lagrangian (3.4) is invariant in the bulk under supersymmetry of the superPoincaré group. Then, in the rheonomic approach, we have that $\imath_{\epsilon}\left(d \mathcal{L}_{\text {bulk }}\right)=0$ is satisfied. Nevertheless, the supersymmetry invariance of the Lagrangian is not guaranteed when a boundary is present. In particular, in presence of a non-trivial boundary, the condition

$$
\left.\imath_{\epsilon} \mathcal{L}\right|_{\partial \mathcal{M}}=0
$$

is not necessarily satisfied and requires to be revised in order to restore supersymmetry invariance of the theory. To this purpose, we have to modify the bulk Lagrangian by adding terms that do not modify the dynamics but affect only the boundary. Thus, we have to consider boundary topological terms.

A good candidate for being considered as a boundary contribution should first scale homogeneously. In particular, each term must have the same scale-weight as the EH term. However, the only boundary terms that can be constructed using the spin-connection $\omega^{a b}$, the vielbein $V^{a}$ and the gravitino $\psi$ are

$$
\begin{aligned}
d\left(\omega^{a b} \mathcal{R}^{c d}+\omega_{f}^{a}{ }_{f} \omega^{f b} \omega^{c d}\right) \epsilon_{a b c d} & =\mathcal{R}^{a b} \mathcal{R}^{c d} \epsilon_{a b c d}, \\
d\left(\bar{\psi} \gamma^{5} \rho\right) & =\bar{\rho} \gamma_{5} \rho
\end{aligned}
$$

which scale with $\omega^{0}$ and $\omega$, respectively. One could add arbitrary constants with appropriate scale-weight but this would imply the presence of a cosmological constant term in the bulk and would reproduce a sum of quadratic terms in the osp (4|1) covariant super field-strengths [33].

An alternative approach is to add new gauge fields with upper scale-weight than the present ones. The minimal content that can be added consists of a bosonic and a fermionic

\footnotetext{
${ }^{3}$ Notice that (3.4) is written as a first-order Lagrangian, and the field equation for the spin-connection $\omega^{a b}$ implies (up to boundary terms, which will be considered subsequently in this work) the vanishing, on-shell, of the supertorsion $R^{a}$ defined in eq. (3.2).
} 
gauge field. In particular, we propose a new antisymmetric bosonic gauge field $A^{a b}=-A^{b a}$ with scale-weight $\omega^{2}$ and an additional fermionic gauge field $\chi$ with scale-weight $\omega^{3 / 2}$. Naturally, one could consider additional bosonic gauge fields with scale-weight $\omega$ and $\omega^{2}$ but, as we shall see, this will not be necessary to recover the supersymmetry invariance in the boundary.

The only boundary contributions constructed by using $\left\{\omega^{a b}, V^{a}, A^{a b}, \psi, \chi\right\}$ that are compatible with parity, Lorentz invariance and that do not involve a scaling parameter are given by the following topological terms:

$$
\begin{aligned}
d\left(A^{a b} \mathcal{R}^{c d}+\omega_{f}^{a} \omega^{f b} A^{c d}+2 \omega_{f}^{a} A^{f b} \omega^{c d}+\omega^{a b} \mathcal{F}^{c d}\right) \epsilon_{a b c d} & =2 \mathcal{R}^{a b} \mathcal{F}^{c d} \epsilon_{a b c d}, \\
d\left(D \bar{\chi} \gamma_{5} \psi+D \bar{\psi} \gamma_{5} \chi\right) & =2 \bar{\sigma} \gamma_{5} \rho+\frac{1}{4} \mathcal{R}^{a b} \bar{\chi} \gamma^{c d} \psi \epsilon_{a b c d},
\end{aligned}
$$

where we have defined $\sigma \equiv D \chi$ and $\mathcal{F}^{a b} \equiv D A^{a b}$ as the respective covariant derivatives of the new gauge fields. Thus, the boundary Lagrangian reads

$$
\mathcal{L}_{\text {bdy }}=\alpha\left(2 \bar{\sigma} \gamma_{5} \rho+\frac{1}{4} R^{a b} \bar{\chi} \gamma^{c d} \psi \epsilon_{a b c d}\right)+\beta\left(2 \mathcal{R}^{a b} \mathcal{F}^{c d} \epsilon_{a b c d}\right)
$$

where $\alpha$ and $\beta$ are constant parameters. Note that the boundary Lagrangian has scaleweight $\omega^{2}$ as the bulk Lagrangian.

Then, we have the following full Lagrangian:

$$
\begin{aligned}
\mathcal{L}_{\text {full }}= & \mathcal{L}_{\text {bulk }}+\mathcal{L}_{\text {bdy }} \\
= & \epsilon_{a b c d} \mathcal{R}^{a b} V^{c} V^{d}+4 \bar{\psi} V^{a} \gamma_{a} \gamma_{5} \rho \\
& +\alpha\left(2 \bar{\sigma} \gamma_{5} \rho+\frac{1}{4} \mathcal{R}^{a b} \bar{\chi} \gamma^{c d} \psi \epsilon_{a b c d}\right)+\beta\left(2 \mathcal{R}^{a b} \mathcal{F}^{c d} \epsilon_{a b c d}\right) .
\end{aligned}
$$

The supersymmetry invariance of the full Lagrangian requires

$$
\delta_{\epsilon} \mathcal{L}_{\text {full }} \equiv l_{\epsilon} \mathcal{L}_{\text {full }}=\imath_{\epsilon} d \mathcal{L}_{\text {full }}+d\left(\imath_{\epsilon} \mathcal{L}_{\text {full }}\right)=0
$$

Naturally, the boundary terms (3.11) that we have introduced do not affect the bulk and the supersymmetry invariance in the bulk is still satisfied such that $\imath_{\epsilon} d \mathcal{L}_{\text {full }}=0$. Then, the supersymmetry invariance of the full Lagrangian $\mathcal{L}_{\text {full }}$ requires to verify the condition $\left.\imath_{\epsilon}\left(\mathcal{L}_{\text {full }}\right)\right|_{\partial \mathcal{M}}=0$. In particular, we have

$$
\begin{aligned}
\imath_{\epsilon}\left(\mathcal{L}_{\text {full }}\right)= & \epsilon_{a b c d} \imath_{\epsilon}\left(\mathcal{R}^{a b}\right) V^{c} V^{d}+4 \bar{\epsilon} V^{a} \gamma_{a} \gamma_{5} \rho+4 \bar{\psi} V^{a} \gamma_{a} \gamma_{5} \imath_{\epsilon}(\rho) \\
& +\alpha\left\{2 \imath_{\epsilon}(\bar{\sigma}) \gamma_{5} \rho+2 \bar{\sigma} \gamma_{5} \imath_{\epsilon}(\rho)+\frac{1}{4}\left[\imath_{\epsilon}\left(\mathcal{R}^{a b}\right) \bar{\chi} \gamma^{c d} \psi+\mathcal{R}^{a b} \bar{\chi} \gamma^{c d} \epsilon\right] \epsilon_{a b c d}\right\} \\
& +2 \beta\left[\imath_{\epsilon}\left(\mathcal{R}^{a b}\right) \mathcal{F}^{c d} \epsilon_{a b c d}+\mathcal{R}^{a b} \imath_{\epsilon}\left(\mathcal{F}^{c d}\right) \epsilon_{a b c d}\right] .
\end{aligned}
$$

In general, $\imath_{\epsilon} \mathcal{L}_{\text {full }}$ is not zero; however, its projection on the boundary should be zero. One can see that the field equations acquire non-trivial boundary contributions coming not only from the boundary Lagrangian but also from the bulk Lagrangian (from the total 
differentials originating from partial integration), which implies the following constraints on the boundary:

$$
\left\{\begin{aligned}
\left.\mathcal{R}^{a b}\right|_{\partial \mathcal{M}} & =0 \\
\left.\mathcal{F}^{a b}\right|_{\partial \mathcal{M}} & =-\frac{1}{2 \beta}\left(V^{a} V^{b}+\frac{\alpha}{4} \bar{\chi} \gamma^{a b} \psi\right)_{\partial \mathcal{M}} \\
\left.\rho\right|_{\partial \mathcal{M}} & =0 \\
\left.\sigma\right|_{\partial \mathcal{M}} & =-\frac{2}{\alpha}\left(V^{a} \gamma_{a} \psi\right)_{\partial \mathcal{M}} .
\end{aligned}\right.
$$

Thus, the super curvatures on the boundary result to be fixed to constant values in an enlarged anholonomic basis (indeed, on the boundary they are fixed in terms of not only the supervielbein, but also of the extra 1-form field $\chi$ ). Nevertheless, as we will see in the sequel, their rheonomic parametrization results to be well defined in ordinary superspace. Thus, the boundary values of the super curvatures in superspace are dynamically fixed by the field equations of the full Lagrangian (understood as bulk plus boundary contributions).

Upon use of (3.16) we find that

$$
\left.\imath_{\epsilon}\left(\mathcal{L}_{\text {full }}\right)\right|_{\partial \mathcal{M}}=0 \quad \forall \alpha, \beta .
$$

Thus the supersymmetry invariance of the full Lagrangian is restored in the presence of a non-trivial boundary. The full Lagrangian can be then written in terms of (3.16) à la MacDowell-Mansouri [46] for $\alpha=4$ and $\beta=\frac{1}{2}$,

$$
\mathcal{L}_{\text {full }}=\mathcal{R}^{a b} F^{c d} \epsilon_{a b c d}+8 \bar{\Xi} \gamma_{5} \rho,
$$

where we have defined

$$
\begin{aligned}
F^{a b} & =\mathcal{F}^{a b}+V^{a} V^{b}+\bar{\chi} \gamma^{a b} \psi, \\
\Xi & =\sigma+\frac{1}{2} V^{a} \gamma_{a} \psi .
\end{aligned}
$$

It is important to point out that the supersymmetry invariance of the flat supergravity Lagrangian on the boundary can be restored by adding new gauge fields enlarging the Poincaré symmetry. In particular, this can be done for arbitrary values of $\alpha$ and $\beta$. Nevertheless, the full Lagrangian à la MacDowell-Mansouri appears for specific values of the constants $\alpha$ and $\beta\left(\alpha=4\right.$ and $\left.\beta=\frac{1}{2}\right)$.

One could think that a dynamical Lagrangian can be recovered from the original MacDowell-Mansouri Lagrangian constructed from the $\operatorname{OSp}(4 \mid 1)$ covariant super curvatures. Although the bulk Lagrangians can be related through a flat limit, the vanishing cosmological constant limit cannot be naively applied in presence of a non-trivial boundary. Indeed, in the flat limit, the only term that remains is a boundary topological term and corresponds to a Gauss-Bonnet term. This is mainly due to the presence of the $\ell$ parameter (length scale) in every term of the bulk Lagrangian of the OSp $(4 \mid 1)$ supergroup. Thus, in order to obtain the pure supergravity action without cosmological constant term using the MacDowell-Mansouri formalism, it is necessary to enlarge the symmetry. This enlargement, as we have shown, does not modify the bulk Lagrangian but affects only the boundary allowing to restore the supersymmetry invariance. 
Let us observe that, interestingly, for $\alpha=4$ and $\beta=\frac{1}{2}$ we also find out that the curvatures (3.16) reproduce the minimal Maxwell covariant super curvatures [47, 51], namely (3.19), (3.20), and

$$
\begin{aligned}
R^{a b} & =\mathcal{R}^{a b}, \\
\Psi & =\rho, \\
R^{a} & =D V^{a}-\frac{1}{2} \bar{\psi} \gamma^{a} \psi,
\end{aligned}
$$

which satisfy the Bianchi identities

$$
\begin{aligned}
D R^{a b} & =0 \\
D F^{a b} & =2 R_{c}^{a} A^{c b}+2 R^{a} V^{b}+\bar{\Xi} \gamma^{a b} \psi-\bar{\chi} \gamma^{a b} \Psi, \\
D \Psi & =\frac{1}{4} R^{a b} \gamma_{a b} \psi \\
D \Xi & =\frac{1}{4} R^{a b} \gamma_{a b} \chi+\frac{1}{2} R^{a} \gamma_{a} \psi-\frac{1}{2} V^{a} \gamma_{a} \Psi \\
D R^{a} & =R_{b}^{a} V^{b}+\bar{\psi} \gamma^{a} \Psi .
\end{aligned}
$$

In particular, the super-Maxwell curvatures vanish at the boundary:

$$
\begin{aligned}
\left.R^{a b}\right|_{\partial \mathcal{M}} & =0, \\
\left.F^{a b}\right|_{\partial \mathcal{M}} & =0, \\
\left.\Psi\right|_{\partial \mathcal{M}} & =0, \\
\left.\Xi\right|_{\partial \mathcal{M}} & =0 .
\end{aligned}
$$

Then, the full MacDowell-Mansouri like Lagrangian (3.18) can be rewritten as

$$
\mathcal{L}_{\text {full }}=R^{a b} F^{c d} \epsilon_{a b c d}+8 \bar{\Xi} \gamma_{5} \Psi
$$

in terms of the Maxwell super curvatures (3.19), (3.20), and (3.21).

Let us observe that the full Lagrangian obtained here cannot be directly obtained as a flat limit of the $\operatorname{OSp}(4 \mid 1)$ one [33] due to the presence of new gauge fields. Nevertheless, one could apply our approach to the supergravity Lagrangian in presence of the cosmological constant (and thus of a length scale $\ell$ ) by adding the extra gauge fields not only to the boundary Lagrangian but also to the bulk Lagrangian, such that the flat limit $\ell \rightarrow \infty$ reproduces the full Lagrangian (3.18) obtained here.

Before studying the supersymmetry invariance of a supergravity theory with a generalized cosmological constant in the presence of a non-trivial boundary, let us provide the rheonomic parametrization of the Maxwell super curvatures and the supersymmetry transformation laws. 


\subsection{Maxwell supersymmetry}

The minimal Maxwell superalgebra has been introduced in [47] in order to describe a generalized four-dimensional superspace in the presence of a constant abelian supersymmetric field-strength background. Such superalgebra has the particularity to extend the Maxwell symmetry $\left\{J_{a b}, P_{a}, Z_{a b}\right\}$ by adding two spinorial generators $\left\{Q_{\alpha}, \Sigma_{\alpha}\right\}$. In particular, the generators satisfy the following non-vanishing (anti)commutation relations:

$$
\begin{aligned}
{\left[J_{a b}, J_{c d}\right] } & =\eta_{b c} J_{a d}-\eta_{a c} J_{b d}-\eta_{b d} J_{a c}+\eta_{a d} J_{b c}, \\
{\left[J_{a b}, P_{c}\right] } & =\eta_{b c} P_{a}-\eta_{a c} P_{b}, \\
{\left[P_{a}, P_{b}\right] } & =Z_{a b}, \\
{\left[J_{a b}, Z_{c d}\right] } & =\eta_{b c} Z_{a d}-\eta_{a c} Z_{b d}-\eta_{b d} Z_{a c}+\eta_{a d} Z_{b c}, \\
{\left[J_{a b}, Q_{\alpha}\right] } & =-\frac{1}{2}\left(\gamma_{a b} Q\right)_{\alpha}, \\
{\left[J_{a b}, \Sigma_{\alpha}\right] } & =-\frac{1}{2}\left(\gamma_{a b} \Sigma\right)_{\alpha}, \\
{\left[P_{a}, Q_{\alpha}\right] } & =-\frac{1}{2}\left(\gamma_{a} \Sigma\right)_{\alpha}, \\
\left\{Q_{\alpha}, Q_{\beta}\right\} & =\left(\gamma^{a} C\right)_{\alpha \beta} P_{a}, \\
\left\{Q_{\alpha}, \Sigma_{\beta}\right\} & =-\frac{1}{2}\left(\gamma^{a b} C\right)_{\alpha \beta} Z_{a b} .
\end{aligned}
$$

Concerning the purely bosonic level, the Maxwell algebra has been introduced in $[52,53]$. Such symmetry and its generalizations have been recently useful to recover General Relativity from Chern-Simons (CS) and Born-Infeld (BI) gravity formalisms [54-58]. More recently, there has been a new interest in exploring the three-dimensional Maxwell CS gravity [59-63]. At the supersymmetric level, the super-Maxwell family also appears in three spacetime dimensions allowing to reproduce CS supergravity models [64-66]. Further generalizations of the minimal Maxwell superalgebra can be found in [67-72] with diverse applications.

In the geometric approach, the most general ansatz for the super-Maxwell curvatures in the supervielbein basis $\left\{V^{a}, \psi\right\}$ of superspace is given by

$$
\begin{aligned}
R^{a b} & =R^{a b}{ }_{c d} V^{c} \wedge V^{d}+\bar{\Theta}^{a b}{ }_{c} \psi \wedge V^{c}+\lambda_{1} \bar{\psi} \wedge \gamma^{a b} \psi, \\
R^{a} & =R_{b c}^{a}{ }_{b c} V^{b} \wedge V^{c}+\bar{\Theta}^{a}{ }_{b} \psi \wedge V^{b}+\lambda_{2} \bar{\psi} \wedge \gamma^{a} \psi, \\
F^{a b} & =F^{a b}{ }_{c d} V^{c} \wedge V^{d}+\bar{\Lambda}^{a b}{ }_{c} \psi \wedge V^{c}+\lambda_{3} \bar{\psi} \wedge \gamma^{a b} \psi, \\
\Psi & =\Psi_{a b} V^{a} \wedge V^{b}+\lambda_{4} \gamma_{a} \psi \wedge V^{a}+\Omega_{\alpha \beta} \psi^{\alpha} \wedge \psi^{\beta}, \\
\Xi & =\Xi_{a b} V^{a} \wedge V^{b}+\lambda_{5} \gamma_{a} \psi \wedge V^{a}+\tilde{\Omega}_{\alpha \beta} \psi^{\alpha} \wedge \psi^{\beta},
\end{aligned}
$$

where $\bar{\Theta}^{a b}{ }_{c}, \bar{\Theta}^{a}{ }_{b}, \bar{\Lambda}^{a b}{ }_{c}$ are spinor-tensors, $\Omega_{\alpha \beta}, \tilde{\Omega}_{\alpha \beta}$ are Majorana spinor-valued matrices, and the $\lambda_{i}$ 's $(i=1,2, \ldots, 5)$ constant parameters. Considering the on-shell condition $R^{a}=$ 0 and studying the various sectors of the on-shell Bianchi identities (3.22) in superspace, 
one can show that the full set of super curvatures are parameterized as follows:

$$
\begin{aligned}
R^{a b} & =R^{a b}{ }_{c d} V^{c} V^{d}+\bar{\Theta}^{a b}{ }_{c} \psi V^{c}, \\
R^{a} & =0, \\
F^{a b} & =F^{a b}{ }_{c d} V^{c} V^{d}+\bar{\Lambda}^{a b}{ }_{c} \psi V^{c}, \\
\Psi & =\Psi_{a b} V^{a} V^{b}, \\
\Xi & =\lambda_{5} \gamma_{a} \psi V^{a},
\end{aligned}
$$

with

$$
\bar{\Theta}_{c}^{a b}=\epsilon^{a b d e}\left(\bar{\Psi}_{c d} \gamma_{e} \gamma_{5}+\bar{\Psi}_{e c} \gamma_{d} \gamma_{5}-\bar{\Psi}_{d e} \gamma_{c} \gamma_{5}\right)
$$

Such parametrization allows us to obtain explicitly the supersymmetry transformation laws. In particular, in the rheonomic approach, a 1-form superfield transformation on spacetime takes the form

$$
\delta \mu^{A}=(\nabla \epsilon)^{A}+\imath_{\epsilon} R^{A},
$$

where $\epsilon^{A}$ are the gauge parameters and $R^{A}$ are the parameterized super curvatures. Then, restricting us to supersymmetry transformation and considering the parametrization (3.27) of the super-Maxwell curvatures, we find the following supersymmetry transformation laws:

$$
\begin{aligned}
\delta_{\epsilon} \omega^{a b} & =2 \bar{\Theta}^{a b}{ }_{c} \epsilon V^{c}, \\
\delta_{\epsilon} V^{a} & =\bar{\epsilon} \gamma^{a} \psi, \\
\delta_{\epsilon} A^{a b} & =\bar{\xi} \gamma^{a b} \epsilon+2 \bar{\Lambda}^{a b}{ }_{c} \epsilon V^{c}, \\
\delta_{\epsilon} \psi & =D \epsilon \\
\delta_{\epsilon} \chi & =V^{a} \gamma_{a} \epsilon
\end{aligned}
$$

where we have set $\lambda_{5}=-\frac{1}{2}$.

\section{Supergravity with generalized cosmological constant in presence of a non-trivial boundary}

The presence of additional bosonic generators has been recently related to a generalization of the cosmological constant $[35,51,73,74]$. Nevertheless, as we have previously seen in section 3 , the introduction of additional gauge fields does not necessary imply the presence of a cosmological constant term in a (super)gravity action. In order to include a cosmological constant contribution to our supergravity model, it is necessary to switch on an 
explicit scale. In particular, we can write the following super curvatures: ${ }^{4}$

$$
\begin{aligned}
\mathfrak{R}^{a b} & \equiv d \omega^{a b}+\omega_{c}^{a} \omega^{c b}+\frac{1}{2 \ell} \bar{\psi} \gamma^{a b} \psi, \\
\mathfrak{R}^{a} & \equiv D V^{a}+\frac{1}{\ell^{2}} A_{b}^{a} V^{b}-\frac{1}{2} \bar{\psi} \gamma^{a} \psi-\frac{1}{\ell} \bar{\psi} \gamma^{a} \chi-\frac{1}{2 \ell^{2}} \bar{\chi} \gamma^{a} \chi, \\
\rho & \equiv D \psi, \\
\mathfrak{F}^{a b} & \equiv D A^{a b}+V^{a} V^{b}+\bar{\chi} \gamma^{a b} \psi+\frac{1}{\ell^{2}} A_{c}^{a} A^{c b}+\frac{1}{2 \ell} \bar{\chi} \gamma^{a b} \chi, \\
\Omega & \equiv D \chi+\frac{1}{2} V^{a} \gamma_{a} \psi+\frac{1}{2 \ell} V^{a} \gamma_{a} \chi+\frac{1}{4 \ell} A^{a b} \gamma_{a b} \psi+\frac{1}{4 \ell^{2}} A^{a b} \gamma_{a b} \chi,
\end{aligned}
$$

being, as usual, $D=d+\omega$ the Lorentz covariant derivative. The next step is the explicit construction of the bulk Lagrangian.

\subsection{Rheonomic construction of the bulk Lagrangian}

The most general ansatz for the geometric bulk Lagrangian can be written as

$$
\mathcal{L}=\mu^{(4)}+R^{A} \mu_{A}^{(2)}+R^{A} R^{B} \mu_{A B}^{(0)},
$$

where the upper index $(p)$ denotes the degree of the related $p$-forms. Here, the $R^{A}$ 's are the super curvatures defined by eq. (4.1), which are invariant under the following rescaling:

$$
\omega^{a b} \rightarrow \omega^{a b}, \quad V^{a} \rightarrow \omega V^{a}, \quad A^{a b} \rightarrow \omega^{2} A^{a b}, \quad \psi \rightarrow \omega^{1 / 2} \psi, \quad \chi \rightarrow \omega^{3 / 2} \chi .
$$

In particular, the Lagrangian scales with $\omega^{2}$, being $\omega^{2}$ the scale-weight of the EH term. On the other hand, since we are interested in the construction of the bulk Lagrangian, we can set $R^{A} R^{B} \mu_{A B}^{(0)}=0$, since terms of this type correspond to boundary contributions. Then, considering the explicit form of the super curvatures (4.1) and applying the parity conservation law, we are left with the following Lagrangian:

$$
\begin{aligned}
\mathcal{L}= & \epsilon_{a b c d} \Re^{a b} V^{c} V^{d}+\frac{\alpha_{1}}{\ell^{2}} \epsilon_{a b c d} \mathfrak{F}^{a b} V^{c} V^{d}+\alpha_{2} \bar{\psi} \gamma_{a} \gamma_{5} \rho V^{a}+\frac{\alpha_{3}}{\ell} \bar{\psi} \gamma_{a} \gamma_{5} \Omega V^{a} \\
& +\frac{\alpha_{4}}{\ell^{2}} \bar{\chi} \gamma_{a} \gamma_{5} \Omega V^{a}+\frac{\alpha_{5}}{\ell} \bar{\chi} \gamma_{a} \gamma_{5} \rho V^{a}+\frac{\alpha_{6}}{\ell^{2}} \epsilon_{a b c d} V^{a} V^{b} V^{c} V^{d}+\frac{\alpha_{7}}{\ell} \epsilon_{a b c d} \bar{\psi} \gamma^{a b} \psi V^{c} V^{d} \\
& +\frac{\alpha_{8}}{\ell^{2}} \epsilon_{a b c d} \bar{\psi} \gamma^{a b} \chi V^{c} V^{d}+\frac{\alpha_{9}}{\ell^{3}} \epsilon_{a b c d} \bar{\chi} \gamma^{a b} \chi V^{c} V^{d}
\end{aligned}
$$

where we have set the coefficient of the first term in (4.4) to 1 . The $\alpha_{i}$ 's constants are dimensionless parameters which can be determined from the equations of motion. Indeed, considering the variation of the Lagrangian with respect to the gauge fields we obtain the following field equations:

$$
\begin{aligned}
& \delta_{\omega} \mathcal{L}=\delta_{A} \mathcal{L}=0 \rightarrow \epsilon_{a b c d} \Re^{c} V^{d}=0 \\
& \delta_{V} \mathcal{L}=0 \rightarrow 2 \epsilon_{a b c d}\left(\mathfrak{R}^{a b}+\frac{1}{\ell^{2}} \mathfrak{F}^{a b}\right) V^{c}+4\left(\bar{\psi}+\frac{1}{\ell} \chi\right) \gamma_{d} \gamma_{5} \rho+\frac{4}{\ell}\left(\bar{\psi}+\frac{1}{\ell} \bar{\chi}\right) \gamma_{d} \gamma_{5} \Omega=0, \\
& \delta_{\psi} \mathcal{L}=\delta_{\chi} \mathcal{L}=0 \rightarrow 8 V^{a} \gamma_{a} \gamma_{5}\left(\rho+\frac{1}{\ell} \Omega\right)+4 \gamma_{a} \gamma_{5}\left(\psi+\frac{1}{\ell} \chi\right) \mathfrak{R}^{a}=0
\end{aligned}
$$

\footnotetext{
${ }^{4}$ Notice that these super curvatures can be obtained by considering the same scale-weight for the gauge fields as in the flat case. The main difference with the previous curvatures consists in the explicit presence of the length parameter $\ell$.
} 
where, in order to recover consistent field equations involving the (generalized) curvature two-forms (4.1), we have set

$$
\begin{aligned}
& \alpha_{1}=1, \\
& \alpha_{2}=\alpha_{3}=\alpha_{4}=\alpha_{5}=4, \\
& \alpha_{6}=\alpha_{7}=\alpha_{9}=-\frac{1}{2}, \\
& \alpha_{8}=-1 .
\end{aligned}
$$

In particular, setting $\alpha_{1}=1$ and $\alpha_{2}=\alpha_{3}=\alpha_{4}=\alpha_{5}=4, \delta_{\omega} \mathcal{L}=0$ reproduces the field equation for the generalized supertorsion, that is $\epsilon_{a b c d} \Re^{c} V^{d}=0$ (thus, the first equation corresponds to the vanishing of the generalized supertorsion $\mathfrak{R}^{a}$ ). The variation of the Lagrangian with respect to the gauge field $A^{a b}$ yields the same result. On the other hand, the curvatures $\mathfrak{R}^{a b}$ and $\mathfrak{F}^{a b}$ appear explicitly in the generalized equation of motion obtained when varying the Lagrangian with respect to the vielbein $V^{a}$ by setting $\alpha_{6}=\alpha_{7}=\alpha_{9}=-\frac{1}{2}$ and $\alpha_{8}=-1$. Finally, from the variation of the Lagrangian with respect to the gravitino $\psi$ (as well as from the variation with respect to $\chi$ ) one obtains the generalized equation involving the curvature 2 -forms $\rho$ and $\Omega$ (together with the generalized supertorsion $\mathfrak{R}^{a}$ ). Let us observe that the equations of motion (4.5) are a generalization of the $\operatorname{OSp}(4 \mid 1)$ ones, the only differences being related to the presence of the new fields $A^{a b}$ and $\chi$ (and of their respective super field-strength). Moreover, the equations of motion obtained by varying the Lagrangian with respect to the new fields $A^{a b}$ and $\chi$ are just a double of the field equations obtained from the variation with respect to the spin-connection $\omega^{a b}$ and the gravitino $\psi$, respectively. With these particular values of the constants, the equations of motion of the Lagrangian admit an AdS vacuum solution with a generalized cosmological constant given by $\frac{1}{\ell^{2}} \epsilon_{a b c d} \mathfrak{F}^{a b} V^{c} V^{d}-\frac{1}{2 \ell^{2}} \epsilon_{a b c d} V^{a} V^{b} V^{c} V^{d}$, where $\mathfrak{F}^{a b}$ has been defined in (4.1). As was noticed in [73], the presence of an additional gauge field in gravity allows to define a generalized cosmological constant term written in terms of the vielbein $V^{a}$ and the new gauge field $A^{a b}$. Such feature was then extended to supergravity in [35, 51, 74], offering, in particular, an alternative way of introducing a generalized cosmological term in this context. It is interesting to note that flat supergravity with boundary could appear from a supergravity action in presence of a more general cosmological term.

Thus, the bulk Lagrangian can be rewritten in terms of Lorentz-type curvatures as follows:

$$
\begin{aligned}
\mathcal{L}_{\mathrm{bulk}}= & \epsilon_{a b c d} R^{a b} V^{c} V^{d}+\frac{1}{\ell^{2}} \epsilon_{a b c d} \mathbb{F}^{a b} V^{c} V^{d}+4 \bar{\psi} \gamma_{a} \gamma_{5} \rho V^{a}+\frac{4}{\ell} \bar{\psi} \gamma_{a} \gamma_{5} \Phi V^{a} \\
& +\frac{4}{\ell^{2}} \bar{\chi} \gamma_{a} \gamma_{5} \Phi V^{a}+\frac{4}{\ell} \bar{\chi} \gamma_{a} \gamma_{5} \rho V^{a}+\frac{1}{2 \ell^{2}} \epsilon_{a b c d} V^{a} V^{b} V^{c} V^{d} \\
& +\frac{1}{\ell} \epsilon_{a b c d} \bar{\psi} \gamma^{a b} \psi V^{c} V^{d}+\frac{2}{\ell^{2}} \epsilon_{a b c d} \bar{\chi} \gamma^{a b} \psi V^{c} V^{d}+\frac{1}{\ell^{3}} \epsilon_{a b c d} \bar{\chi} \gamma^{a b} \chi V^{c} V^{d},
\end{aligned}
$$


with

$$
\begin{aligned}
R^{a b} & =d \omega^{a b}+\omega_{c}^{a} \omega^{c b}, \\
\mathfrak{R}^{a} & =D V^{a}+\frac{1}{\ell^{2}} A_{b}^{a} V^{b}-\frac{1}{2} \bar{\psi} \gamma^{a} \psi-\frac{1}{\ell} \bar{\psi} \gamma^{a} \chi-\frac{1}{2 \ell^{2}} \bar{\chi} \gamma^{a} \chi, \\
\rho & =D \psi \\
\mathbb{F}^{a b} & =D A^{a b}+\frac{1}{\ell^{2}} A_{c}^{a} A^{c b}, \\
\Phi & =D \chi+\frac{1}{4 \ell} A^{a b} \gamma_{a b} \psi+\frac{1}{4 \ell^{2}} A^{a b} \gamma_{a b} \chi .
\end{aligned}
$$

Let us note that the presence of the length parameter $\ell$ in the super curvatures allows to introduce, in an alternative way, a generalized supersymmetric cosmological constant term in the Lagrangian. Such Lagrangian can be seen as a deformation of the usual supergravity Lagrangian for the $\mathfrak{o s p}(4 \mid 1)$ superalgebra. Interestingly, as for the case of the $\mathfrak{o s p}(4 \mid 1)$ supergravity, the vanishing cosmological constant limit $\ell \rightarrow \infty$ leads to the flat supergravity Lagrangian. However, as we have discussed in section 3, the supersymmetry invariance of flat supergravity on a manifold with boundary is recovered by adding non-trivial boundary (topological) terms. Then, it seems that the flat limit in presence of a boundary requires to consider a deformation of the full Lagrangian for the $\mathfrak{o s p}(4 \mid 1)$ superalgebra. Thus, the bulk Lagrangian (4.7) obtained here seems a good candidate to consider in presence of a boundary. Observe that, when an explicit scale is switched on, terms involving the new 1-form fields $A^{a b}$ and $\chi$ are allowed to appear in the bulk Lagrangian, which was not the case for flat supergravity, where, however, as we have previously shown, they play a crucial role in restoring the supersymmetry invariance in the presence of a non-trivial boundary.

Before studying the explicit boundary contributions required to assure supersymmetry invariance we shall provide the supersymmetry transformation laws under which the bulk Lagrangian (4.7) is invariant.

The supersymmetry transformations can be obtained from the rheonomic parametrization of the super curvatures which are determined from the study of the Bianchi identities of the Lorentz-type curvatures (4.8). In particular, the super curvatures (4.8) fulfill the following Bianchi identities:

$$
\begin{aligned}
D R^{a b} & =0 \\
D \Re^{a} & =R_{b}^{a} V^{b}+\frac{1}{\ell^{2}} \mathbb{F}_{b}^{a} V^{a}-\frac{1}{\ell^{2}} A_{b}^{a} \Re^{a}+\bar{\psi} \gamma^{a} \rho+\frac{1}{\ell} \bar{\chi} \gamma^{a} \rho+\frac{1}{\ell} \bar{\psi} \gamma^{a} \Phi+\frac{1}{\ell^{2}} \bar{\chi} \gamma^{a} \Phi, \\
D \rho & =\frac{1}{4} R^{a b} \gamma_{a b} \psi \\
D \mathbb{F}^{a b} & =2 R_{c}^{a} A^{c b}+\frac{2}{\ell^{2}} \mathbb{F}_{c}^{a} A^{c b} \\
D \Phi & =\frac{1}{4} R^{a b} \gamma_{a b} \chi+\frac{1}{4 \ell} \mathbb{F}^{a b} \gamma_{a b} \psi-\frac{1}{4 \ell} A^{a b} \gamma_{a b} \rho+\frac{1}{4 \ell^{2}} \mathbb{F}^{a b} \gamma_{a b} \chi-\frac{1}{4 \ell^{2}} A^{a b} \gamma_{a b} \Phi .
\end{aligned}
$$

Notice that the super curvatures above are defined in a superspace larger than the ordinary one. In the following, we will ask the curvatures parametrization to be well defined in ordinary superspace by exploiting the rheonomic approach. One can show that the 
Bianchi identities (4.9) are satisfied by parameterizing (on-shell) the super curvatures in the following way:

$$
\begin{aligned}
R^{a b} & =\mathcal{R}^{a b}{ }_{c d} V^{c} V^{d}+\bar{\Pi}^{a b}{ }_{c} \psi V^{c}+\varsigma_{1} \bar{\psi} \gamma^{a b} \psi, \\
\mathfrak{R}^{a} & =0, \\
\mathbb{F}^{a b} & =\mathcal{F}^{a b}{ }_{c d} V^{c} V^{d}+\bar{\Delta}^{a b}{ }_{c} \psi V^{c}, \\
\rho & =\rho_{a b} V^{a} V^{b}+\varsigma_{2} \gamma_{a} \psi V^{a}, \\
\Phi & =\Phi_{a b} V^{a} V^{b}+\varsigma_{3} \gamma_{a} \psi V^{a},
\end{aligned}
$$

where

$$
\begin{aligned}
\bar{\Pi}^{a b}{ }_{c} & =\epsilon^{a b d e}\left(\bar{\rho}_{c d} \gamma_{e} \gamma_{5}+\bar{\rho}_{e c} \gamma_{d} \gamma_{5}-\bar{\rho}_{d e} \gamma_{c} \gamma_{5}\right), \\
\bar{\Delta}^{a b}{ }_{c} & =\epsilon^{a b d e}\left(\bar{\Phi}_{c d} \gamma_{e} \gamma_{5}+\bar{\Phi}_{e c} \gamma_{d} \gamma_{5}-\bar{\Phi}_{d e} \gamma_{c} \gamma_{5}\right), \\
\varsigma_{1} & =-\varsigma_{2}-\frac{1}{\ell} \varsigma_{3},
\end{aligned}
$$

and where we have set $\mathfrak{R}^{a}=0$ (on-shell condition). Such parametrization of the super curvatures in ordinary superspace provides us with the supersymmetry transformation laws for the 1-form gauge fields, which read as follows:

$$
\begin{aligned}
\delta_{\epsilon} \omega^{a b} & =2 \bar{\Pi}^{a b}{ }_{c} \epsilon V^{c}+2 \varsigma_{1} \bar{\epsilon} \gamma^{a b} \psi, \\
\delta_{\epsilon} V^{a} & =\bar{\epsilon} \gamma^{a} \psi-\frac{1}{\ell} \bar{\chi} \gamma^{a} \epsilon, \\
\delta_{\epsilon} A^{a b} & =2 \bar{\Delta}^{a b}{ }_{c} \epsilon V^{c}, \\
\delta_{\epsilon} \psi & =D \epsilon+\varsigma_{2} \gamma_{a} \epsilon V^{a}, \\
\delta_{\epsilon} \chi & =\varsigma_{3} \gamma_{a} \epsilon V^{a} .
\end{aligned}
$$

It is important to point out that the spacetime Lagrangian (4.7) is invariant up to boundary terms under the supersymmetry transformations (4.12) of the gauge fields on spacetime. If the spacetime background has a non-trivial boundary, we have to check explicitly the supersymmetry invariance.

\subsection{Supersymmetry invariance in presence of a boundary}

Let us now consider the supergravity theory previously introduced in presence of a nontrivial spacetime boundary, and let us study the supersymmetry invariance of it. In particular, we shall see that appropriate boundary terms are required in order to restore the supersymmetry invariance of the full Lagrangian given by the bulk Lagrangian (4.7) plus boundary contributions. Although the supersymmetry invariance in the bulk is satisfied, the invariance of the Lagrangian when a boundary is present, is not trivially satisfied:

$$
\left.\imath_{\epsilon} \mathcal{L}_{\text {bulk }}\right|_{\partial \mathcal{M}} \neq 0 .
$$


Then, for recovering the supersymmetry invariance of the theory, it is necessary to modify the bulk Lagrangian by adding suitable boundary terms. The only possible boundary contributions compatible with parity and Lorentz invariance are given by

$$
\begin{aligned}
& d\left(\omega^{a b} R^{c d}+\omega_{f}^{a} \omega^{f b} \omega^{c d}\right) \epsilon_{a b c d}=\epsilon_{a b c d} R^{a b} R^{c d} \\
& d\left(A^{a b} R^{c d}+\omega_{f}^{a} \omega^{f b} A^{c d}\right.+2 \omega_{f}^{a} A^{f b} \omega^{c d}+\omega^{a b} \mathbb{F}^{c d}+\frac{1}{\ell^{2}} A_{f}^{a} A^{f b} \omega^{c d}+\frac{2}{\ell^{2}} \omega_{f}^{a} A^{f b} A^{c d} \\
&\left.\frac{1}{\ell^{2}} A^{a b} \mathbb{F}^{c d}+\frac{1}{\ell^{4}} A_{f}^{a} A^{f b} A^{c d}\right) \epsilon_{a b c d}=\epsilon_{a b c d}\left(2 R^{a b} \mathbb{F}^{c d}+\frac{1}{\ell^{2}} \mathbb{F}^{a b} \mathbb{F}^{c d}\right) \\
& d\left(\bar{\psi} \gamma_{5} \rho\right)=\bar{\rho} \gamma_{5} \rho+\frac{1}{8} R^{a b} \bar{\psi} \gamma^{c d} \psi \epsilon_{a b c d} \\
& d\left(\bar{\psi} \gamma_{5} \Phi+\bar{\chi} \gamma_{5} \rho+\frac{1}{\ell} \bar{\chi} \gamma_{5} \Phi\right)=2 \bar{\rho} \gamma_{5} \Phi+\bar{\Phi} \gamma_{5} \Phi+\frac{1}{4} R^{a b} \bar{\psi} \gamma^{c d} \chi \epsilon_{a b c d}+\frac{1}{8 \ell} \mathbb{F}^{a b} \bar{\psi} \gamma^{c d} \psi \epsilon_{a b c d} \\
&+\frac{1}{4 \ell^{2}} \mathbb{F}^{a b} \bar{\psi} \gamma^{c d} \chi \epsilon_{a b c d}+\frac{1}{8 \ell} R^{a b} \bar{\chi} \gamma^{c d} \chi \epsilon_{a b c d}+\frac{1}{8 \ell^{3}} \mathbb{F}^{a b} \bar{\chi} \gamma^{c d} \chi \epsilon_{a b c d} .
\end{aligned}
$$

The boundary terms (4.14) allow us to write the following boundary Lagrangian:

$$
\begin{aligned}
\mathcal{L}_{\mathrm{bdy}}= & \lambda \epsilon_{a b c d} R^{a b} R^{c d}+\pi\left(\bar{\rho} \gamma_{5} \rho+\frac{1}{8} R^{a b} \bar{\psi} \gamma^{c d} \psi \epsilon_{a b c d}\right)+\mu \epsilon_{a b c d}\left(2 R^{a b} \mathbb{F}^{c d}+\frac{1}{\ell^{2}} \mathbb{F}^{a b} \mathbb{F}^{c d}\right) \\
& +\nu\left(2 \bar{\rho} \gamma_{5} \Phi+\bar{\Phi} \gamma_{5} \Phi+\frac{1}{4} R^{a b} \bar{\psi} \gamma^{c d} \chi \epsilon_{a b c d}+\frac{1}{8 \ell} \mathbb{F}^{a b} \bar{\psi} \gamma^{c d} \psi \epsilon_{a b c d}\right. \\
& \left.+\frac{1}{4 \ell^{2}} \mathbb{F}^{a b} \bar{\psi} \gamma^{c d} \chi \epsilon_{a b c d}+\frac{1}{8 \ell} R^{a b} \bar{\chi} \gamma^{c d} \chi \epsilon_{a b c d}+\frac{1}{8 \ell^{3}} \mathbb{F}^{a b} \bar{\chi} \gamma^{c d} \chi \epsilon_{a b c d}\right)
\end{aligned}
$$

where $\lambda, \pi, \mu, \nu$ are constant parameters. In order to have a consistently defined limit $\ell \rightarrow \infty$ (flat limit) at the level of the full Lagrangian, one should drop out the terms involving positive powers of $\ell$. From the Lagrangian (4.15) we see that all terms have scaleweight $\omega^{2}$ except those proportional to $\lambda$ and $\pi$. Thus, in order to have an appropriately scaled boundary Lagrangian one should define new (dimensionless) constants, $\lambda^{\prime}$ and $\pi^{\prime}$, such that $\lambda=\ell^{2} \lambda^{\prime}$ and $\pi=\ell \pi^{\prime}$, which implies positive powers of $\ell$. Then, the terms proportional to $\lambda$ and $\pi$ must be dropped out.

In this way, we are left with

$$
\begin{aligned}
\mathcal{L}_{\mathrm{bdy}}= & \mu \epsilon_{a b c d}\left(2 R^{a b} \mathbb{F}^{c d}+\frac{1}{\ell^{2}} \mathbb{F}^{a b} \mathbb{F}^{c d}\right) \\
& +\nu\left(2 \bar{\rho} \gamma_{5} \Phi+\bar{\Phi} \gamma_{5} \Phi+\frac{1}{4} R^{a b} \bar{\psi} \gamma^{c d} \chi \epsilon_{a b c d}+\frac{1}{8 \ell} \mathbb{F}^{a b} \bar{\psi} \gamma^{c d} \psi \epsilon_{a b c d}\right. \\
& \left.+\frac{1}{4 \ell^{2}} \mathbb{F}^{a b} \bar{\psi} \gamma^{c d} \chi \epsilon_{a b c d}+\frac{1}{8 \ell} R^{a b} \bar{\chi} \gamma^{c d} \chi \epsilon_{a b c d}+\frac{1}{8 \ell^{3}} \mathbb{F}^{a b} \bar{\chi} \gamma^{c d} \chi \epsilon_{a b c d}\right)
\end{aligned}
$$


Thus, let us consider the following full Lagrangian:

$$
\begin{aligned}
\mathcal{L}_{\text {full }}= & \mathcal{L}_{\text {bulk }}+\mathcal{L}_{\text {bdy }} \\
= & \epsilon_{a b c d} R^{a b} V^{c} V^{d}+\frac{1}{\ell^{2}} \epsilon_{a b c d} \mathbb{F}^{a b} V^{c} V^{d}+4 \bar{\psi} \gamma_{a} \gamma_{5} \rho V^{a} \\
& +\frac{4}{\ell} \bar{\psi} \gamma_{a} \gamma_{5} \Phi V^{a}+\frac{4}{\ell^{2}} \bar{\chi} \gamma_{a} \gamma_{5} \Phi V^{a}+\frac{4}{\ell} \bar{\chi} \gamma_{a} \gamma_{5} \rho V^{a} \\
& +\frac{1}{2 \ell^{2}} \epsilon_{a b c d} V^{a} V^{b} V^{c} V^{d}+\frac{1}{\ell} \epsilon_{a b c d} \bar{\psi} \gamma^{a b} \psi V^{c} V^{d}+\frac{2}{\ell^{2}} \epsilon_{a b c d} \bar{\chi} \gamma^{a b} \psi V^{c} V^{d} \\
& +\frac{1}{\ell^{3}} \epsilon_{a b c d} \bar{\chi} \gamma^{a b} \chi V^{c} V^{d}+\mu \epsilon_{a b c d}\left(2 R^{a b} \mathbb{F}^{c d}+\frac{1}{\ell^{2}} \mathbb{F}^{a b} \mathbb{F}^{c d}\right) \\
& +\nu\left(2 \bar{\rho} \gamma_{5} \Phi+\frac{1}{\ell} \bar{\Phi} \gamma_{5} \Phi+\frac{1}{4} R^{a b} \bar{\psi} \gamma^{c d} \chi \epsilon_{a b c d}+\frac{1}{8 \ell} \mathbb{F}^{a b} \bar{\psi} \gamma^{c d} \psi \epsilon_{a b c d}\right. \\
& \left.+\frac{1}{4 \ell^{2}} \mathbb{F}^{a b} \bar{\psi} \gamma^{c d} \chi \epsilon_{a b c d}+\frac{1}{8 \ell} R^{a b} \bar{\chi} \gamma^{c d} \chi \epsilon_{a b c d}+\frac{1}{8 \ell^{3}} \mathbb{F}^{a b} \bar{\chi} \gamma^{c d} \chi \epsilon_{a b c d}\right) .
\end{aligned}
$$

Now we have to verify the supersymmetry invariance of the full Lagrangian. Clearly, $\imath_{\epsilon} d \mathcal{L}_{\text {full }}=0$, since the boundary terms are total derivatives. Then, considering the condition

$$
\delta_{\epsilon} \mathcal{L}_{\text {full }}=\imath_{\epsilon} d \mathcal{L}_{\text {full }}+d\left(\imath_{\epsilon} \mathcal{L}_{\text {full }}\right)=0,
$$

we have just to verify that $\left.\imath_{\epsilon}\left(\mathcal{L}_{\text {full }}\right)\right|_{\partial \mathcal{M}}=0$ is satisfied on the boundary. Considering (4.17), we have

$$
\begin{aligned}
\imath_{\epsilon}\left(\mathcal{L}_{\text {full }}\right)= & \epsilon_{a b c d} \imath_{\epsilon}\left(R^{a b}+\frac{1}{\ell^{2}} \mathbb{F}^{a b}\right) V^{c} V^{d}+4 \bar{\epsilon} V^{a} \gamma_{a} \gamma_{5} \rho+4 \bar{\psi} V^{a} \gamma_{a} \gamma_{5} \imath_{\epsilon}(\rho) \\
& +\frac{4}{\ell} \bar{\epsilon} V^{a} \gamma_{a} \gamma_{5} \Phi+\frac{4}{\ell} \bar{\psi} V^{a} \gamma_{a} \gamma_{5} \imath_{\epsilon}(\Phi)+\frac{4}{\ell^{2}} \bar{\chi} V^{a} \gamma_{a} \gamma_{5} \imath_{\epsilon}(\Phi) \\
& +\frac{4}{\ell} \bar{\chi} V^{a} \gamma_{a} \gamma_{5} \imath_{\epsilon}(\rho)+\left(\frac{2}{\ell} \bar{\epsilon} \gamma^{a b} \psi V^{c} V^{d}+\frac{2}{\ell^{2}} \epsilon_{a b c d} \bar{\chi} \gamma^{a b} \epsilon V^{c} V^{d}\right) \epsilon_{a b c d} \\
& +\mu \epsilon_{a b c d} \imath_{\epsilon}\left(2 R^{a b}+\frac{1}{\ell^{2}} \mathbb{F}^{a b}\right) \mathbb{F}^{c d}+\mu \epsilon_{a b c d}\left(2 R^{a b}+\frac{1}{\ell^{2}} \mathbb{F}^{a b}\right) \imath_{\epsilon}\left(\mathbb{F}^{c d}\right) \\
& +2 \nu\left[\imath_{\epsilon}(\bar{\rho}) \gamma_{5} \Phi+\bar{\rho} \gamma_{5} \imath_{\epsilon}(\Phi)+\frac{1}{\ell} \imath_{\epsilon}(\bar{\Phi}) \gamma_{5} \Phi\right] \\
& +\nu \epsilon_{a b c d}\left[\frac{1}{4} \imath_{\epsilon}\left(R^{a b}+\frac{1}{\ell^{2}} \mathbb{F}^{a b}\right) \bar{\psi} \gamma^{c d} \chi+\frac{1}{4}\left(R^{a b}+\frac{1}{\ell^{2}} \mathbb{F}^{a b}\right) \bar{\epsilon} \gamma^{c d} \chi\right. \\
& \left.+\frac{1}{8 \ell} \imath_{\epsilon}\left(R^{a b}+\frac{1}{\ell^{2}} \mathbb{F}^{a b}\right) \bar{\chi} \gamma^{c d} \chi+\frac{1}{8 \ell} \imath_{\epsilon}\left(\mathbb{F}^{a b}\right) \bar{\psi} \gamma^{c d} \psi+\frac{1}{4 \ell} \mathbb{F}^{a b} \bar{\epsilon} \gamma^{c d} \psi\right]
\end{aligned}
$$

Then, one can prove that $\left.\frac{\delta \mathcal{L}_{\text {full }}}{\delta \mu^{A}}\right|_{\partial \mathcal{M}}=0$ leads to the following constraints on the boundary:

$$
\left\{\begin{aligned}
\left.R^{a b}\right|_{\partial \mathcal{M}} & =-\frac{\nu}{16 \mu \ell}\left(\bar{\psi} \gamma^{a b} \psi\right)_{\partial \mathcal{M}} \\
\left.\mathbb{F}^{a b}\right|_{\partial \mathcal{M}} & =-\frac{1}{2 \mu}\left(V^{a} V^{b}+\frac{\nu}{4} \bar{\chi} \gamma^{a b} \psi+\frac{\nu}{8 \ell} \bar{\chi} \gamma^{a b} \chi\right)_{\partial \mathcal{M}} \\
\left.\rho\right|_{\partial \mathcal{M}} & =0 \\
\left.\Phi\right|_{\partial \mathcal{M}} & =-\frac{2}{\nu}\left(V^{a} \gamma_{a} \psi+\frac{1}{\ell} V^{a} \gamma_{a} \chi\right)_{\partial \mathcal{M}}
\end{aligned}\right.
$$


Again, the super curvatures on the boundary result to be fixed to constant values in an enlarged anholonomic basis (given by the supervielbein together with the 1-form field $\chi$ ). Nevertheless, as we have explicitly shown in (4.10), the rheonomic parametrization of these super curvatures results to be well defined in ordinary superspace.

Then, upon use of (4.20) we get

$$
\left.\imath_{\epsilon}\left(\mathcal{L}_{\text {full }}\right)\right|_{\partial \mathcal{M}}=0 \Leftrightarrow \frac{\nu}{8 \mu}+\frac{4}{\nu}=2 .
$$

This condition can be written as

$$
\nu=8 \mu(1+h), \quad h^{2}=1-\frac{1}{2 \mu} ; \quad(\nu \neq 0 \Rightarrow h \neq-1) .
$$

One can see that setting $h=0$, we obtain

$$
\mu=\frac{1}{2} \Rightarrow \nu=4
$$

Remarkably, with these values for $\mu$ and $\nu$, the full Lagrangian (4.17) can be written à la MacDowell-Mansouri as

$$
\mathcal{L}_{\text {full }}=\mathfrak{R}^{a b} \mathfrak{F}^{c d} \epsilon_{a b c d}+\frac{1}{2 \ell^{2}} \mathfrak{F}^{a b} \mathfrak{F}^{c d} \epsilon_{a b c d}+8 \bar{\Omega} \gamma_{5} \rho+\frac{4}{\ell} \bar{\Omega} \gamma_{5} \Omega,
$$

written exactly in terms of the curvatures (4.1).

We have thus shown that the supersymmetric boundary terms given in (4.15) allow to recover the supersymmetry invariance of our supergravity model in the presence of a non-trivial boundary. One can notice, using (4.20), that the super curvatures (4.1) vanish at the boundary.

The flat limit $\ell \rightarrow \infty$ is now well defined in the MacDowell-Mansouri formalism. Indeed, unlike the case of the full Lagrangian obtained for osp $(4 \mid 1)$ supergravity in the presence of a non-trivial boundary [33], the vanishing cosmological constant limit of the full Lagrangian (4.24) reproduces appropriately the flat supergravity model with boundary that we have considered initially. In particular, in this case not only the bulk Lagrangians are well related through the flat limit but also the boundary contributions. Furthermore, one can see that the super-Maxwell curvatures (3.19), (3.20), and (3.21) are recovered as a flat limit of the super curvatures (4.1). It is then natural to expect that the minimal Maxwell superalgebra (3.25) appears as a flat limit of a deformation of the $\mathfrak{o s p}(4 \mid 1)$ superalgebra.

\subsubsection{AdS-Lorentz supersymmetry}

The (anti)commutation relations of the superalgebra related to the super curvatures (4.1) are given by

$$
\begin{aligned}
{\left[J_{a b}, J_{c d}\right] } & =\eta_{b c} J_{a d}-\eta_{a c} J_{b d}-\eta_{b d} J_{a c}+\eta_{a d} J_{b c} \\
{\left[J_{a b}, Z_{c d}\right] } & =\eta_{b c} Z_{a d}-\eta_{a c} Z_{b d}-\eta_{b d} Z_{a c}+\eta_{a d} Z_{b c} \\
{\left[Z_{a b}, Z_{c d}\right] } & =\frac{1}{\ell^{2}}\left(\eta_{b c} Z_{a d}-\eta_{a c} Z_{b d}-\eta_{b d} Z_{a c}+\eta_{a d} Z_{b c}\right) \\
{\left[J_{a b}, P_{c}\right] } & =\eta_{b c} P_{a}-\eta_{a c} P_{b}, \quad\left[Z_{a b}, P_{c}\right]=\frac{1}{\ell^{2}}\left(\eta_{b c} P_{a}-\eta_{a c} P_{b}\right), \\
{\left[P_{a}, P_{b}\right] } & =Z_{a b}
\end{aligned}
$$




$$
\begin{array}{rlrl}
{\left[J_{a b}, Q_{\alpha}\right]} & =-\frac{1}{2}\left(\gamma_{a b} Q\right)_{\alpha}, & {\left[J_{a b}, \Sigma_{\alpha}\right]=-\frac{1}{2}\left(\gamma_{a b} \Sigma\right)_{\alpha},} \\
{\left[Z_{a b}, Q_{\alpha}\right]} & =-\frac{1}{2 \ell}\left(\gamma_{a b} \Sigma\right)_{\alpha}, & {\left[Z_{a b}, \Sigma_{\alpha}\right]=-\frac{1}{2 \ell^{2}}\left(\gamma_{a b} \Sigma\right)_{\alpha},} \\
{\left[P_{a}, Q_{\alpha}\right]} & =-\frac{1}{2}\left(\gamma_{a} \Sigma\right)_{\alpha}, & {\left[P_{a}, \Sigma_{\alpha}\right]=-\frac{1}{2 \ell}\left(\gamma_{a} \Sigma\right)_{\alpha},} \\
\left\{Q_{\alpha}, Q_{\beta}\right\} & =-\frac{1}{2 \ell}\left(\gamma^{a b} C\right)_{\alpha \beta} J_{a b}+\left(\gamma^{a} C\right)_{\alpha \beta} P_{a}, \\
\left\{Q_{\alpha}, \Sigma_{\beta}\right\} & =-\frac{1}{2}\left(\gamma^{a b} C\right)_{\alpha \beta} Z_{a b}+\frac{1}{\ell}\left(\gamma^{a} C\right)_{\alpha \beta} P_{a}, \\
\left\{\Sigma_{\alpha}, \Sigma_{\beta}\right\} & =-\frac{1}{2 \ell}\left(\gamma^{a b} C\right)_{\alpha \beta} Z_{a b}+\frac{1}{\ell^{2}}\left(\gamma^{a} C\right)_{\alpha \beta} P_{a},
\end{array}
$$

with $a=0,1,2,3, \alpha=1,2,3,4$, and where the generators $J_{a b}, P_{a}, Z_{a b}, Q$ and $\Sigma$ are respectively dual to the 1 -form fields $\omega^{a b}, V^{a}, A^{a b}, \psi$, and $\chi$. Interestingly, the present superalgebra corresponds to an alternative supersymmetric extension of the AdS-Lorentz symmetry (given by eq. (4.25)). Such bosonic algebra, also known as a semisimple extension of the Poincaré symmetry, was first presented in [48, 49]. Then, it was generalized to a family of $\mathfrak{C}_{k}$ algebras $[75,76]$ which have been useful to recover diverse (pure) Lovelock theories from CS and BI gravity theories [77-79].

Although it contains two spinorial charges as the minimal AdS-Lorentz superalgebras introduced in [51], the superalgebra given by eqs. (4.25) and (4.26) does not require additional bosonic generators with respect to the ones of the AdS-Lorentz algebra in order to satisfy the Jacobi identities. The closure of this superalgebra is guaranteed by the explicit form of the anticommutators in (4.26). Indeed, any subtle deformation of the aforementioned anticommutators requires to introduce additional bosonic generators as in $[40,51,80]$. On the other hand, it is important to signalize that the supersymmetrization of the AdS-Lorentz algebra is not unique. In particular, a super AdS-Lorentz symmetry with one spinor charge has also been considered in [48, 81, 82].

Note that in the limit $\ell \rightarrow \infty$ the above superalgebra gives exactly the super-Maxwell algebra (3.25), without any auxiliary generator.

As a final remark, it is important to point out that in [35] a supergravity model in presence of a non-trivial boundary for the AdS-Lorentz superalgebra with one spinor charge has been presented. Nevertheless, such construction does not allow for a proper flat limit. In particular, although it is possible to recover a non-standard Maxwell superalgebra from the super AdS-Lorentz one considered in [35], the exotic anticommutation relation of the non-standard Maxwell does not allow a proper construction of a supergravity action. Such problematic comes from the fact that the four-momentum generators no longer appear as a result of the anticommutator of the fermionic generators. It seems that, as we have shown here, the only possibility to have a well defined vanishing cosmological constant limit for the AdS-Lorentz supergravity is to consider an additional fermionic charge. Remarkably, such flat limit allows to recover the usual flat supergravity with non-trivial boundary contributions. 


\section{Discussion}

In this paper, we have studied the supersymmetry invariance of flat supergravity in presence of a non-trivial boundary. We have shown that supersymmetry invariance is achieved by adding proper topological terms in which additional gauge fields are considered. We have found that the full Lagrangian can be rewritten à la MacDowell-Mansouri in terms of Maxwell super curvatures. Interestingly, the bulk Lagrangian corresponds to the usual flat supergravity Lagrangian. Although the extra fields only appear in the boundary contributions, they are essential to recover supersymmetry invariance of the full Lagrangian. Furthermore, as the topological term in pure gravity allows to regularize the action, one could argue that the presence of the new gauge fields in the boundary would allow to regularize the supergravity action in the holographic renormalization language.

We have also explored the possibility of introducing a well-defined vanishing cosmological constant limit in a supergravity model in order to recover the Lagrangian obtained in the case of flat spacetime with a non-trivial boundary. As we have discussed, flat supergravity with boundary cannot be naively obtained from $\mathfrak{o s p}(4 \mid 1)$ supergravity with boundary (MacDowell-Mansouri action). Indeed, this require to consider an enlarged supergravity with a generalized cosmological constant which has been obtained using the rheonomic approach. We have shown that, as in the flat case, supersymmetry invariance requires to add appropriate topological terms. The full supergravity obtained within this procedure can be rewritten in terms of the super curvatures of a particular AdS-Lorentz superalgebra. Remarkably, the flat limit of the full Lagrangian properly reproduces flat supergravity on a manifold with boundary.

To our knowledge, this is the first report showing that the supersymmetry invariance of a supergravity theory on a manifold with boundary is restored by adding new gauge fields in the boundary. However, the introduction of new gauge fields which enlarge the symmetry group has been already considered in the literature with different purposes. Of particular interest are the Maxwell and AdS-Lorentz algebras. Although such symmetries have been particularly useful to relate diverse gravity theories [54-58, 77-79], the dynamical implication of considering an enlarged set of fields has been poorly explored. In early works, in order to interpret the Maxwell (super)algebra, and the corresponding (super)group, a Maxwell-invariant particle model on the extended spacetime was studied [49, 80, 83-86]. In particular, it was shown that the interaction term described by a Maxwell-invariant 1-form introduces new tensor degrees of freedom that, in the equations of motion, play the role of a background electromagnetic field which is constant on-shell and leads to a closed, Maxwell-invariant 2-form. On the other hand, in [73] the Maxwell algebra was exploited in order introduce the cosmological term in Einstein gravity in an alternative way. This appeared in a generalized form, with a dependence on the additional gauge fields $A_{\mu}^{a b}$ associated with the generators $Z_{a b}$, giving an interpretation of the Maxwell algebra in the context of inflation, since the latter can also be driven by suitably coupled vector fields. Such feature was then extended to supergravity in [35, 51, 74], offering, in particular, an alternative way of introducing a generalized cosmological term in this context. 
Moreover, recently it was shown in three spacetime dimensions that the presence of the extra fields in the Chern-Simons action invariant under the Maxwell algebra influences the vacuum energy and the vacuum angular momentum of the stationary configuration [63]. In addition, it has been shown that the boundary dynamics of such gravity theory is described by an enlargement and deformation of the $\mathfrak{b m s}_{3}$ algebra with three central charges. On the other hand, considering suitable boundary conditions, a semi-simple enlargement of the $\mathfrak{b m s}_{3}$ algebra appears as the asymptotic symmetry at null infinity of a Chern-Simons gravity action based on the AdS-Lorentz algebra [87]. The extension of these results to supergravity remains unknown and it would be interesting to generalize the study in four dimensions.

Furthermore, as we have already mentioned, the presence of an extra (that is besides $Q$ ) fermionic generator (we called it $\Sigma$ in the present work) dual to a spinor 1-form (here, $\chi$ ) is naturally involved in the minimal supersymmetrization of the Maxwell algebra. On the other hand, other relevant superalgebras containing two fermionic charges were introduced and deeply analyzed in [71, 72, 88-91]: precisely, the D'Auria-Fré superalgebra, introduced in [88] and subsequently further analyzed in [90, 91], underlying the Free Differential Algebra of $D=11$ supergravity, the Green algebra [89], in the context of superstring, and Maxwell-type superalgebras recently shown to be related to $D=4$ and $D=11$ supergravity [71, 72]. Then, we conjecture the existence of possible relations among the new 1-form fields $A^{a b}$ and $\chi$ appearing in our model (and, eventually, in extensions to higher dimensions) and the extra fields appearing in the aforementioned theories. This could shed some light on the physical and group theoretical role played by $A^{a b}$ and $\chi$. Concerning supergravity theories, an interesting relation has already been found in [72] (following previous studies presented for the first time in [88] and further analyzed in [71, 90, 91]), where it was shown that the Maxwell superalgebra in four dimensions, whose corresponding super curvatures are the ones given in (3.19), (3.20), and (3.21), can be interpreted as a hidden superalgebra underlying $\mathcal{N}=1, D=4$ supergravity extended to include a 2-form gauge potential associated to a 2-index antisymmetric tensor (in this scenario, the theory is appropriately discussed in the context of Free Differential Algebras, an extension of the Maurer-Cartan equations to involve higher-degree differential forms). The same extra spinor dual to the nilpotent fermionic generator whose presence is crucial for writing a supersymmetric extension of the Maxwell algebra turned out to be fundamental ingredients also to reproduce the $D=4$ Free Differential Algebra on ordinary superspace. A future work on this side would consist in extending the analysis to higher dimensions, clarifying, in this context, the physical (and topological) role of the field $A^{a b}$, dual to the almost-central (in the sense that it commutes with all the superalgebra but the Lorentz generators), bosonic generator $Z_{a b}$ that could be understood as a 2-brane charge (probably under a generalized perspective), source of a 3 -form gauge potential. Furthermore, the study could be extended to the case in which an explicit scale is switched on.

It is important to observe that, as in $[33,35,40]$, the full supergravity Lagrangian can be rewritten à la MacDowell-Mansouri. This would suggest, as it was pointed out in the bosonic case [92, 93], a superconformal structure which is an interesting additional motivation to our study. On the other hand, our particular approach in which we consider 
additional gauge fields in the boundary could be useful in order to explore the supersymmetry invariance of supergravities with boundary coupled to matter or in higher dimensions. Indeed, it would be interesting to explore the boundary contributions required to recover supersymmetry invariance of a general matter coupled $\mathcal{N}=2$ supergravity in four dimensions [94, 95]. Naturally, one could consider first a supergravity theory coupled to scalar field. The extension of our results to $\mathcal{N}=2$ is more subtle and will be studied in a future work.

Finally, let us highlight that in the present work we have shown that the supersymmetry invariance of a supergravity theory on a manifold with boundary is restored by adding new gauge fields in the boundary, concentrating just on the four-dimensional theory to show that the supersymmetry of the four-dimensional Lagrangian given by bulk plus boundary contributions can indeed be recovered. In this context, the supersymmetry invariance of the $D=4$ theory is achieved without requiring Dirichlet boundary conditions on the fields at the boundary; rather we have found that the boundary values of the super curvatures are dynamically fixed to constant values in an enlarged anholonomic basis (indeed, on the boundary they are fixed in terms of not only the supervielbein, but also of the extra 1-form field $\chi$ ). Nevertheless, as we have explicitly shown, the rheonomic parametrization of these super curvatures results to be well defined in ordinary superspace.

However, here we shall mention that what commonly happens is that the insertion of a spacetime boundary breaks a part of the boundary supersymmetries, and thus one would expect that our boundary conditions break a part of the bulk supersymmetries on the boundary. The analysis of the symmetry breaking pattern in this context would require the study of the theory living on the boundary and, in particular, the explicit three-dimensional description of the equations (precisely, of the boundary conditions on the super field-strength) we have obtained in $D=4$. Thus, one could investigate on the boundary theory produced in our framework: in order to discuss the theory living on the boundary (in the spirit of the holographic correspondence) one should set the boundary at $r \rightarrow \infty$, where $r$ is the radial coordinate, and study the asymptotic limit $r \rightarrow \infty$ of the $D=3$ equations on the boundary (in particular, one should properly choose the boundary behavior of the $D=4$ fields which relates them to the $D=3$ ones and perform the asymptotic limit $r \rightarrow \infty$ ). The explicit three-dimensional description of the equations we have obtained in $D=4$ would depend on the general symmetry properties of the theory on the boundary.

In the scenario of our work, where we have the presence of a non-trivial boundary of spacetime (meaning that the boundary is not thought as set at infinity and thus the fields do not asymptotically vanish) and of extra bosonic and fermionic 1-form fields ( $A^{a b}$ and $\chi$, respectively), we conjecture that the related boundary theory (at least, concerning the enlarged supergravity model involving the scale parameter $\ell$; then, one could further study the limit $\ell \rightarrow \infty$ in this context) could feature some generalization of deformed locally $\mathrm{AdS}_{3}$ geometries, due to the presence of extra $D=4$ fields from the very beginning.

Moreover, we conjecture that such an analysis could also shed further light on the study done in [39], where the authors found unexpected intriguing relations between $\mathcal{N}=2$, $D=4$ supergravity and a three-dimensional theory describing the properties of graphene 
and featuring "unconventional local supersymmetry". Besides, it would be interesting to further discuss our results in light of the ones obtained in [96] in the context of flat holography and Carrollian fluids, where the authors demonstrated, using the derivative expansion of fluid/gravity correspondence, that a holographic description of four-dimensional asymptotically locally flat spacetimes is reached smoothly from the zero cosmological constant limit of AdS holography. Intriguingly, from the boundary perspective the vanishing of the bulk cosmological constant appears as the zero velocity of light limit, setting how Carrollian geometry emerges in flat holography. The analysis of the boundary theory produced in our framework, together with a deeper investigation on the role played by the spinor 1-form field $\chi$ and the bosonic 1-form field $A^{a b}$ appearing in our model in this context, will be will the subject of a future work.

\section{Acknowledgments}

The authors wish to thank L. Andrianopoli, R. D'Auria and M. Trigiante for enlightening discussions and their kind hospitality at DISAT of Politecnico di Torino (Italy), where the main discussion of this work was done. This work was supported by the Chilean FONDECYT Projects N$^{\circ} 3170437$ (P.C.) and $N^{\circ} 3170438$ (E.R.).

Open Access. This article is distributed under the terms of the Creative Commons Attribution License (CC-BY 4.0), which permits any use, distribution and reproduction in any medium, provided the original author(s) and source are credited.

\section{References}

[1] J.W. York, Jr., Role of conformal three geometry in the dynamics of gravitation, Phys. Rev. Lett. 28 (1972) 1082 [INSPIRE].

[2] G.W. Gibbons and S.W. Hawking, Action Integrals and Partition Functions in Quantum Gravity, Phys. Rev. D 15 (1977) 2752 [InSPIRE].

[3] J.D. Brown and J.W. York, Jr., Quasilocal energy and conserved charges derived from the gravitational action, Phys. Rev. D 47 (1993) 1407 [gr-qc/9209012] [INSPIRE].

[4] P. Hořava and E. Witten, Eleven-dimensional supergravity on a manifold with boundary, Nucl. Phys. B 475 (1996) 94 [hep-th/9603142] [INSPIRE].

[5] J.M. Maldacena, The Large $N$ limit of superconformal field theories and supergravity, Int. J. Theor. Phys. 38 (1999) 1113 [hep-th/9711200] [INSPIRE].

[6] S.S. Gubser, I.R. Klebanov and A.M. Polyakov, Gauge theory correlators from noncritical string theory, Phys. Lett. B 428 (1998) 105 [hep-th/9802109] [INSPIRE].

[7] E. Witten, Anti-de Sitter space and holography, Adv. Theor. Math. Phys. 2 (1998) 253 [hep-th/9802150] [INSPIRE].

[8] O. Aharony, S.S. Gubser, J.M. Maldacena, H. Ooguri and Y. Oz, Large N field theories, string theory and gravity, Phys. Rept. 323 (2000) 183 [hep-th/9905111] [INSPIRE].

[9] E. D'Hoker and D.Z. Freedman, Supersymmetric gauge theories and the AdS /CFT correspondence, in Strings, Branes and Extra Dimensions: TASI 2001: Proceedings, pp. 3-158, 2002, hep-th/0201253 [INSPIRE]. 
[10] G. Compere and D. Marolf, Setting the boundary free in AdS/CFT, Class. Quant. Grav. 25 (2008) 195014 [arXiv:0805.1902] [INSPIRE].

[11] A.J. Amsel and G. Compère, Supergravity at the boundary of AdS supergravity, Phys. Rev. D 79 (2009) 085006 [arXiv:0901.3609] [InSPIRE].

[12] V. Balasubramanian and P. Kraus, A stress tensor for Anti-de Sitter gravity, Commun. Math. Phys. 208 (1999) 413 [hep-th/9902121] [INSPIRE].

[13] J. de Boer, E.P. Verlinde and H.L. Verlinde, On the holographic renormalization group, JHEP 08 (2000) 003 [hep-th/9912012] [INSPIRE].

[14] E.P. Verlinde and H.L. Verlinde, $R G$ flow, gravity and the cosmological constant, JHEP 05 (2000) 034 [hep-th/9912018] [inSPIRE].

[15] J. de Boer, The Holographic renormalization group, Fortsch. Phys. 49 (2001) 339 [hep-th/0101026] [INSPIRE].

[16] S. de Haro, S.N. Solodukhin and K. Skenderis, Holographic reconstruction of space-time and renormalization in the AdS/CFT correspondence, Commun. Math. Phys. 217 (2001) 595 [hep-th/0002230] [INSPIRE].

[17] K. Skenderis, Lecture notes on holographic renormalization, Class. Quant. Grav. 19 (2002) 5849 [hep-th/0209067] [INSPIRE].

[18] A. Lawrence and A. Sever, Holography and renormalization in Lorentzian signature, JHEP 10 (2006) 013 [hep-th/0606022] [INSPIRE].

[19] I. Heemskerk and J. Polchinski, Holographic and Wilsonian Renormalization Groups, JHEP 06 (2011) 031 [arXiv:1010.1264] [INSPIRE].

[20] D. Astefanesei, N. Banerjee and S. Dutta, (Un)attractor black holes in higher derivative AdS gravity, JHEP 11 (2008) 070 [arXiv:0806.1334] [INSPIRE].

[21] Y. Brihaye and E. Radu, Black objects in the Einstein-Gauss-Bonnet theory with negative cosmological constant and the boundary counterterm method, JHEP 09 (2008) 006 [arXiv:0806.1396] [INSPIRE].

[22] A. Anabalón, D. Astefanesei, D. Choque and C. Martínez, Trace Anomaly and Counterterms in Designer Gravity, JHEP 03 (2016) 117 [arXiv:1511.08759] [INSPIRE].

[23] D. Astefanesei, R. Ballesteros, D. Choque and R. Rojas, Scalar charges and the first law of black hole thermodynamics, Phys. Lett. B 782 (2018) 47 [arXiv:1803.11317] [INSPIRE].

[24] G. Esposito, A. Yu. Kamenshchik and K. Kirsten, One loop effective action for Euclidean Maxwell theory on manifolds with boundary, Phys. Rev. D 54 (1996) 7328 [hep-th/9606132] [INSPIRE].

[25] I.G. Moss, Boundary terms for eleven-dimensional supergravity and M-theory, Phys. Lett. B 577 (2003) 71 [hep-th/0308159] [INSPIRE].

[26] P. van Nieuwenhuizen and D.V. Vassilevich, Consistent boundary conditions for supergravity, Class. Quant. Grav. 22 (2005) 5029 [hep-th/0507172] [INSPIRE].

[27] D.V. Belyaev, Boundary conditions in supergravity on a manifold with boundary, JHEP 01 (2006) 047 [hep-th/0509172] [INSPIRE].

[28] P. van Nieuwenhuizen, A. Rebhan, D.V. Vassilevich and R. Wimmer, Boundary terms in supergravity and supersymmetry, Int. J. Mod. Phys. D 15 (2006) 1643 [hep-th/0606075] [INSPIRE]. 
[29] D.V. Belyaev and P. van Nieuwenhuizen, Simple $d=4$ supergravity with a boundary, JHEP 09 (2008) 069 [arXiv:0806.4723] [INSPIRE].

[30] D.V. Belyaev and P. van Nieuwenhuizen, Tensor calculus for supergravity on a manifold with boundary, JHEP 02 (2008) 047 [arXiv:0711.2272] [INSPIRE].

[31] D. Grumiller and P. van Nieuwenhuizen, Holographic counterterms from local supersymmetry without boundary conditions, Phys. Lett. B 682 (2010) 462 [arXiv:0908.3486] [INSPIRE].

[32] P.S. Howe, T.G. Pugh, K.S. Stelle and C. Strickland-Constable, Ectoplasm with an Edge, JHEP 08 (2011) 081 [arXiv:1104.4387] [INSPIRE].

[33] L. Andrianopoli and R. D'Auria, $N=1$ and $N=2$ pure supergravities on a manifold with boundary, JHEP 08 (2014) 012 [arXiv:1405.2010] [INSPIRE].

[34] L. Di Pietro, N. Klinghoffer and I. Shamir, On Supersymmetry, Boundary Actions and Brane Charges, JHEP 02 (2016) 163 [arXiv: 1502.05976] [INSPIRE].

[35] M.C. Ipinza, P.K. Concha, L. Ravera and E.K. Rodríguez, On the Supersymmetric Extension of Gauss-Bonnet like Gravity, JHEP 09 (2016) 007 [arXiv: 1607.00373] [INSPIRE].

[36] D.Z. Freedman, K. Pilch, S.S. Pufu and N.P. Warner, Boundary Terms and Three-Point Functions: An AdS/CFT Puzzle Resolved, JHEP 06 (2017) 053 [arXiv:1611.01888] [INSPIRE].

[37] P. Benetti Genolini, D. Cassani, D. Martelli and J. Sparks, Holographic renormalization and supersymmetry, JHEP 02 (2017) 132 [arXiv:1612.06761] [INSPIRE].

[38] A. Anabalón, D. Astefanesei, A. Gallerati and M. Trigiante, Hairy Black Holes and Duality in an Extended Supergravity Model, JHEP 04 (2018) 058 [arXiv:1712.06971] [INSPIRE].

[39] L. Andrianopoli, B.L. Cerchiai, R. D'Auria and M. Trigiante, Unconventional supersymmetry at the boundary of $A d S_{4}$ supergravity, JHEP 04 (2018) 007 [arXiv: 1801.08081] [INSPIRE].

[40] A. Banaudi and L. Ravera, Generalized AdS-Lorentz deformed supergravity on a manifold with boundary, Eur. Phys. J. Plus 133 (2018) 514 [arXiv:1803.08738] [InSPIRE].

[41] R. Aros, M. Contreras, R. Olea, R. Troncoso and J. Zanelli, Conserved charges for gravity with locally AdS asymptotics, Phys. Rev. Lett. 84 (2000) 1647 [gr-qc/9909015] [INSPIRE].

[42] R. Aros, M. Contreras, R. Olea, R. Troncoso and J. Zanelli, Conserved charges for even dimensional asymptotically AdS gravity theories, Phys. Rev. D 62 (2000) 044002 [hep-th/9912045] [INSPIRE].

[43] P. Mora, R. Olea, R. Troncoso and J. Zanelli, Finite action principle for Chern-Simons AdS gravity, JHEP 06 (2004) 036 [hep-th/0405267] [INSPIRE].

[44] R. Olea, Mass, angular momentum and thermodynamics in four-dimensional Kerr-AdS black holes, JHEP 06 (2005) 023 [hep-th/0504233] [InSPIRE].

[45] D.P. Jatkar, G. Kofinas, O. Mišković and R. Olea, Conformal Mass in AdS gravity, Phys. Rev. D 89 (2014) 124010 [arXiv:1404.1411] [InSPIRE].

[46] S.W. MacDowell and F. Mansouri, Unified Geometric Theory of Gravity and Supergravity, Phys. Rev. Lett. 38 (1977) 739 [Erratum ibid. 38 (1977) 1376] [INSPIRE].

[47] S. Bonanos, J. Gomis, K. Kamimura and J. Lukierski, Maxwell Superalgebra and Superparticle in Constant Gauge Badkgrounds, Phys. Rev. Lett. 104 (2010) 090401 [arXiv:0911.5072] [INSPIRE]. 
[48] D.V. Soroka and V.A. Soroka, Semi-simple extension of the (super)Poincaré algebra, Adv. High Energy Phys. 2009 (2009) 234147 [hep-th/0605251] [INSPIRE].

[49] J. Gomis, K. Kamimura and J. Lukierski, Deformations of Maxwell algebra and their Dynamical Realizations, JHEP 08 (2009) 039 [arXiv:0906.4464] [INSPIRE].

[50] L. Castellani, R. D'Auria and P. Fré, Supergravity and superstrings: A geometric prespective. Vol. 1 and 2, World Scientific, Singapore (1991).

[51] P.K. Concha, E.K. Rodríguez and P. Salgado, Generalized supersymmetric cosmological term in $N=1$ Supergravity, JHEP 08 (2015) 009 [arXiv: 1504.01898] [INSPIRE].

[52] H. Bacry, P. Combe and J.L. Richard, Group-theoretical analysis of elementary particles in an external electromagnetic field. 1. the relativistic particle in a constant and uniform field, Nuovo Cim. A 67 (1970) 267 [INSPIRE].

[53] R. Schrader, The Maxwell group and the quantum theory of particles in classical homogeneous electromagnetic fields, Fortsch. Phys. 20 (1972) 701 [INSPIRE].

[54] J.D. Edelstein, M. Hassaine, R. Troncoso and J. Zanelli, Lie-algebra expansions, Chern-Simons theories and the Einstein-Hilbert Lagrangian, Phys. Lett. B 640 (2006) 278 [hep-th/0605174] [INSPIRE].

[55] F. Izaurieta, E. Rodríguez, P. Minning, P. Salgado and A. Perez, Standard General Relativity from Chern-Simons Gravity, Phys. Lett. B 678 (2009) 213 [arXiv:0905.2187] [INSPIRE].

[56] P.K. Concha, D.M. Peñafiel, E.K. Rodríguez and P. Salgado, Even-dimensional General Relativity from Born-Infeld gravity, Phys. Lett. B 725 (2013) 419 [arXiv:1309.0062] [INSPIRE].

[57] P.K. Concha, D.M. Peñafiel, E.K. Rodríguez and P. Salgado, Chern-Simons and Born-Infeld gravity theories and Maxwell algebras type, Eur. Phys. J. C 74 (2014) 2741 [arXiv: 1402.0023] [INSPIRE].

[58] P.K. Concha, D.M. Peñafiel, E.K. Rodríguez and P. Salgado, Generalized Poincaré algebras and Lovelock-Cartan gravity theory, Phys. Lett. B 742 (2015) 310 [arXiv:1405.7078] [INSPIRE].

[59] P. Salgado, R.J. Szabo and O. Valdivia, Topological gravity and transgression holography, Phys. Rev. D 89 (2014) 084077 [arXiv: 1401.3653] [InSPIRE].

[60] S. Hoseinzadeh and A. Rezaei-Aghdam, $(2+1)$-dimensional gravity from Maxwell and semisimple extension of the Poincaré gauge symmetric models, Phys. Rev. D 90 (2014) 084008 [arXiv: 1402.0320] [INSPIRE].

[61] R. Caroca, P. Concha, O. Fierro, E. Rodríguez and P. Salgado-ReboLledó, Generalized Chern-Simons higher-spin gravity theories in three dimensions, Nucl. Phys. B 934 (2018) 240 [arXiv:1712.09975] [InSPIRE].

[62] L. Avilés, E. Frodden, J. Gomis, D. Hidalgo and J. Zanelli, Non-Relativistic Maxwell Chern-Simons Gravity, JHEP 05 (2018) 047 [arXiv: 1802.08453] [INSPIRE].

[63] P. Concha, N. Merino, O. Mišković, E. Rodríguez, P. Salgado-ReboLledó and O. Valdivia, Asymptotic symmetries of three-dimensional Chern-Simons gravity for the Maxwell algebra, JHEP 10 (2018) 079 [arXiv: 1805. 08834] [INSPIRE].

[64] P.K. Concha, O. Fierro, E.K. Rodríguez and P. Salgado, Chern-Simons supergravity in D $=3$ and Maxwell superalgebra, Phys. Lett. B 750 (2015) 117 [arXiv:1507.02335] [INSPIRE]. 
[65] P.K. Concha, O. Fierro and E.K. Rodríguez, Inönü-Wigner contraction and $D=2+1$ supergravity, Eur. Phys. J. C 77 (2017) 48 [arXiv:1611.05018] [INSPIRE].

[66] P. Concha, D.M. Peñafiel and E. Rodríguez, On the Maxwell supergravity and flat limit in $2+1$ dimensions, Phys. Lett. B 785 (2018) 247 [arXiv:1807.00194] [INSPIRE].

[67] J.A. de Azcarraga, J.M. Izquierdo, J. Lukierski and M. Woronowicz, Generalizations of Maxwell (super)algebras by the expansion method, Nucl. Phys. B 869 (2013) 303 [arXiv:1210.1117] [INSPIRE].

[68] J.A. de Azcarraga and J.M. Izquierdo, Minimal $D=4$ supergravity from the superMaxwell algebra, Nucl. Phys. B 885 (2014) 34 [arXiv: 1403.4128] [INSPIRE].

[69] P.K. Concha and E.K. Rodríguez, Maxwell Superalgebras and Abelian Semigroup Expansion, Nucl. Phys. B 886 (2014) 1128 [arXiv: 1405.1334] [INSPIRE].

[70] P.K. Concha and E.K. Rodríguez, $N=1$ Supergravity and Maxwell superalgebras, JHEP 09 (2014) 090 [arXiv: 1407.4635] [INSPIRE].

[71] D.M. Peñafiel and L. Ravera, On the Hidden Maxwell Superalgebra underlying $D=4$ Supergravity, Fortsch. Phys. 65 (2017) 1700005 [arXiv: 1701.04234] [INSPIRE].

[72] L. Ravera, Hidden role of Maxwell superalgebras in the free differential algebras of $D=4$ and D = 11 supergravity, Eur. Phys. J. C 78 (2018) 211 [arXiv:1801.08860] [INSPIRE].

[73] J.A. de Azcarraga, K. Kamimura and J. Lukierski, Generalized cosmological term from Maxwell symmetries, Phys. Rev. D 83 (2011) 124036 [arXiv:1012.4402] [INSPIRE].

[74] D.M. Peñafiel and L. Ravera, Generalized cosmological term in $D=4$ supergravity from a new AdS-Lorentz superalgebra, Eur. Phys. J. C 78 (2018) 945 [arXiv:1807.07673] [INSPIRE].

[75] P.K. Concha, R. Durka, N. Merino and E.K. Rodríguez, New family of Maxwell like algebras, Phys. Lett. B 759 (2016) 507 [arXiv:1601.06443] [InSPIRE].

[76] R. Durka, Resonant algebras and gravity, J. Phys. A 50 (2017) 145202 [arXiv:1605.00059] [INSPIRE].

[77] P.K. Concha, R. Durka, C. Inostroza, N. Merino and E.K. Rodríguez, Pure Lovelock gravity and Chern-Simons theory, Phys. Rev. D 94 (2016) 024055 [arXiv: 1603.09424] [InSPIRE].

[78] P.K. Concha, N. Merino and E.K. Rodríguez, Lovelock gravities from Born-Infeld gravity theory, Phys. Lett. B 765 (2017) 395 [arXiv:1606. 07083] [INSPIRE].

[79] P. Concha and E. Rodríguez, Generalized Pure Lovelock Gravity, Phys. Lett. B 774 (2017) 616 [arXiv:1708.08827] [INSPIRE].

[80] S. Bonanos, J. Gomis, K. Kamimura and J. Lukierski, Deformations of Maxwell Superalgebras and Their Applications, J. Math. Phys. 51 (2010) 102301 [arXiv:1005.3714] [INSPIRE].

[81] D.V. Soroka and V.A. Soroka, Semi-simple o(N)-extended super-Poincaré algebra, arXiv: 1004.3194 [INSPIRE].

[82] O. Fierro, F. Izaurieta, P. Salgado and O. Valdivia, Minimal AdS-Lorentz supergravity in three-dimensions, Phys. Lett. B 788 (2019) 198 [arXiv:1401.3697] [INSPIRE].

[83] S. Bonanos and J. Gomis, A Note on the Chevalley-Eilenberg Cohomology for the Galilei and Poincaré Algebras, J. Phys. A 42 (2009) 145206 [arXiv:0808.2243] [INSPIRE]. 
[84] S. Bonanos and J. Gomis, Infinite Sequence of Poincaré Group Extensions: Structure and Dynamics, J. Phys. A 43 (2010) 015201 [arXiv:0812.4140] [InSPIRE].

[85] J. Gomis, K. Kamimura and J. Lukierski, Deformed Maxwell Algebras and their Realizations, arXiv:0910.0326 [INSPIRE].

[86] G.W. Gibbons, J. Gomis and C.N. Pope, Deforming the Maxwell-Sim Algebra, Phys. Rev. D 82 (2010) 065002 [arXiv:0910.3220] [InSPIRE].

[87] P. Concha, N. Merino, E. Rodríguez, P. Salgado-ReboLledó and O. Valdivia, Semi-simple enlargement of the $\mathfrak{b m s}_{3}$ algebra from a $\mathfrak{s o}(2,2) \oplus \mathfrak{s o}(2,1)$ Chern-Simons theory, arXiv: 1810.12256 [INSPIRE].

[88] R. D'Auria and P. Fré, Geometric Supergravity in $d=11$ and Its Hidden Supergroup, Nucl. Phys. B 201 (1982) 101 [Erratum ibid. B 206 (1982) 496] [INSPIRE].

[89] M.B. Green, Supertranslations, Superstrings and Chern-Simons Forms, Phys. Lett. B 223 (1989) 157 [INSPIRE].

[90] L. Andrianopoli, R. D'Auria and L. Ravera, Hidden Gauge Structure of Supersymmetric Free Differential Algebras, JHEP 08 (2016) 095 [arXiv: 1606.07328] [INSPIRE].

[91] L. Andrianopoli, R. D'Auria and L. Ravera, More on the Hidden Symmetries of $11 D$ Supergravity, Phys. Lett. B 772 (2017) 578 [arXiv:1705. 06251] [INSPIRE].

[92] O. Mišković and R. Olea, Topological regularization and self-duality in four-dimensional anti-de Sitter gravity, Phys. Rev. D 79 (2009) 124020 [arXiv:0902.2082] [INSPIRE].

[93] O. Mišković, R. Olea and M. Tsoukalas, Renormalized AdS action and Critical Gravity, JHEP 08 (2014) 108 [arXiv:1404.5993] [INSPIRE].

[94] L. Andrianopoli et al., $N=2$ supergravity and $N=2$ superYang-Mills theory on general scalar manifolds: Symplectic covariance, gaugings and the momentum map, J. Geom. Phys. 23 (1997) 111 [hep-th/9605032] [INSPIRE].

[95] L. Andrianopoli, P. Concha, R. D'Auria, E. Rodríguez and M. Trigiante, Observations on BI from $\mathcal{N}=2$ Supergravity and the General Ward Identity, JHEP 11 (2015) 061 [arXiv: 1508.01474] [INSPIRE].

[96] L. Ciambelli, C. Marteau, A.C. Petkou, P.M. Petropoulos and K. Siampos, Flat holography and Carrollian fluids, JHEP 07 (2018) 165 [arXiv: 1802.06809] [INSPIRE]. 